\title{
Die Entwicklung des Lern- und Sozial- verhaltens bei Schülerinnen und Schülern mit Schulassistenz: eine längsschnittliche Studie
}

\author{
Florian Schindler \\ Technische Universität Dortmund
}

\begin{abstract}
Zusammenfassung: Schulassistenz gewinnt im schulischen Kontext zunehmend an Bedeutung, v.a. im Zusammenhang mit schulischer Inklusion. Jedoch ist Schulassistenz sowie insbesondere ihr Einfluss auf die Entwicklung der begleiteten Kinder bislang kaum erforscht. Der vorliegende Beitrag stellt eine längsschnittliche Studie über die Entwicklung des Lern- und Sozialverhaltens von $N=65$ Schüler/innen mit Schulassistenz über den Zeitraum eines Schuljahres hinweg dar. Insgesamt zeigten sich positive Entwicklungen. Unter den erhobenen Teilbereichen erwies sich vor allem die Konzentrationsfähigkeit als positiv verändert, hingegen entwickelte sich die Selbstständigkeit eher negativ. Der Beitrag diskutiert die Ergebnisse bezüglich der Entwicklung des Lern- und Sozialverhaltens bei Schulassistenz differenziert nach unterschiedlichen Altersgruppen und nimmt geschlechtsspezifische Aspekte sowie Bewilligungsstatus und Schultypen in den Blick.
\end{abstract}

Schlüsselbegriffe: Schulassistenz, Integrationshelfer, Lernverhalten, Sozialverhalten, Längsschnitt

\section{Development of Students' Learning and Social Behavior with Respect to Teaching Assistance: A Longitudinal Study}

Summary: School assistance is becoming increasingly important in the context of inclusive education. However, there has been little research into school assistance and its influence on the development of the accompanied children. This paper presents a longitudinal study on the development of the learning and social behaviour of $N=65$ pupils with school assistance over the period of one school year. Overall, positive developments were observed. Among the sub-areas surveyed, the ability to concentrate in particular proved to have changed positively, whereas independence developed rather negatively. The article discusses the results regarding the development of learning and social behaviour in school assistance differentiated according to different age groups. It further looks at gender-specific aspects as well as school types.

Keywords: Paraeducators, teaching assistants, learning behavior, social behavior, longitudinal study

\section{Problemaufriss}

Kinder und Jugendliche haben einen Anspruch auf Teilhabe und Mitwirkung am gesellschaftlichen Leben unabhängig von Erkrankungen, Beeinträchtigungen oder Behinderungen (Oerter \& Höfling, 2001). Dieses Recht auf Teilhabe ist in der UN-Behindertenrechtskonvention
(UN, 2006) festgeschrieben und wird in der Schulpraxis zunehmend unter Einbeziehung von Schulassistent/innen realisiert (Kißgen, Franke, Ladinig, Mays \& Carlitscheck, 2013). So hat innerhalb weniger Jahre eine neue Berufsgruppe Einzug gehalten in Schule und Unterricht - eine Berufsgruppe ohne formale Ausbildung (Meyer, 2017), jedoch mit der höchst 
anspruchsvollen und herausfordernden Aufgabe der besonderen und individuellen Hilfe zu einer angemessenen und begabungsentsprechenden Schulbildung (Heinrich \& Lübeck, 2013; §54 SGB XII).

Während Schulassistenz heute das gesamte Schulsystem - Förderschulen und allgemeine Schulen - betrifft, waren erste Leistungen zur Unterstützung des Schulbesuchs in den 1980er Jahren vor allem an Förderschulen verortet und betrafen Lernende mit schwerer Behinderung und progredienten Erkrankungen (vgl. Dworschak, 2010). Seither haben sich die Tätigkeitsfelder und Konstellationen im Arbeitsfeld Schulassistenz gewandelt: Schulassistenz geht heute über medizinisch-pflegerische Leistungen hinaus und reicht von Maßnahmen zur Unterstützung bei der Alltagsbewältigung über Hilfen auf sozial-emotionaler Ebene bis zur Unterstützung bei Lern- und Unterrichtsvorhaben (vgl. Bacher, Pfaffenberger \& Pöschko, 2007; Beck, Dworschak \& Eibner, 2010; Henn et al., 2014). Bei steigenden Bewilligungszahlen und in einer zunehmend inklusiv arbeitenden Schullandschaft ist Schulassistenz zu einem festen Bestandteil des Schulsystems und zu einer wichtigen Stütze für Lernende und Lehrende geworden.

Die Forschungslage zur Schulassistenz ist derweil noch wenig fundiert. Bereits Dworschak (2010) verweist auf die Problematik, „dass weder ein umfassendes Konzept, noch verlässliche empirische Daten zu dieser Maßnahme vorliegen" (S. 131). Während die Einrichtung einer Schulassistenz in der Praxis stets mit dem Ziel der Unterstützung des Schulbesuchs verbunden ist, wird in der empirischen Forschung gerade die Entwicklung der Leistungsempfänger - der Kinder und Jugendlichen - bislang kaum berücksichtigt. So liegt zum Einfluss von Schulassistenz auf die kindliche Entwicklung im deutschsprachigen Raum bislang lediglich eine Studie vor (Zauner \& Zwosta, 2014), die jedoch - bedingt durch ihr querschnittliches
Design - keine Aussagen über Entwicklungsverläufe von Kindern und Jugendlichen mit Schulassistenz zulässt.

Das Ziel des vorliegenden Beitrags ist es, das Lern- und Sozialverhalten von Schüler/innen mit Schulassistenz über den Zeitraum eines Schuljahres zu untersuchen. Zu diesem Zweck wurde eine Untersuchung mit 82 Klassenlehrkräften durchgeführt, bei der längsschnittlich auswertbare Informationen über 65 Schüler/innen erhoben wurden. Im vorliegenden Beitrag werden die deskriptiven Merkmale und die zeitlichen Veränderungen des Lern- und Sozialverhaltens begleiteter Schüler/innen thematisiert.

\section{Theorie}

Am 26. März 2009 in Deutschland in Kraft getreten, stellt die UN-Behindertenrechtskonvention (UN-BRK) ein Bekenntnis zu einer diskriminierungsfreien und gleichberechtigten Teilhabe aller Menschen unter Anerkennung von Vielfalt und Unterschiedlichkeit dar (UN, 2006). Innerhalb der UN-BRK ist für Kinder und Jugendliche die Umsetzung der schulischen Inklusion von besonderer Bedeutung. Die UNBRK verpflichtet die Vertragsstaaten zu einer chancengleichen und diskriminierungsfreien Bildung. Eine solche Bildung garantiert einen hochwertigen und an den Bedürfnissen des Einzelnen orientierten Unterricht, welcher die erforderlichen Unterstützungsleistungen für einen erfolgreichen Besuch der allgemeinen Schule und eine vollständige Integration umfasst (UN, 2006).

Der Umgang mit Heterogenität und Vielfalt v. a. im Zuge der Inklusion - stellt Schulen vor große organisatorische und unterrichtspraktische Herausforderungen (vgl. z.B. Budde, 2012). Auf Ebene des Unterrichts wird diesen Herausforderungen mit zunehmender Differenzierung und auf Ebene der Rahmenbedingun- 
gen immer häufiger mit den sozialrechtlich verbrieften Leistungen der Eingliederungshilfe begegnet.

\subsection{Schulassistenz - Begriffsklärung}

Die Hilfen zu einer angemessenen Schulbildung stellen unter den Leistungen der Eingliederungshilfe eine der häufigsten Unterstützungsformen für Kinder und Jugendliche dar (Hellrung, 2016). Hierbei handelt es sich um eine Sozialleistung, die entweder im Rahmen der Kinder- und Jugendhilfe (SGB VIII) oder der Sozialhilfe (SGB XII) durchgeführt wird und nach Thiel (2017) mit dem Ziel erfolgt, „den Besuch der Schule und die Teilnahme am Unterricht zu ermöglichen, wenn dieser behinderungsbedingt unter Berücksichtigung der Bedingungen vor Ort gefährdet oder nicht möglich ist" (S. 28f). Realisiert werden diese Leistungen durch den Einsatz von Personen, welche Kinder und Jugendliche im Schulalltag begleiten, „die aufgrund besonderer Bedürfnisse im Kontext Lernen, Verhalten, Kommunikation, Pflege, medizinische Versorgung und/oder Alltagsbewältigung der besonderen und individuellen Unterstützung bei der Verrichtung unterrichtlicher und außerunterrichtlicher Tätigkeiten bedürfen" (Dworschak, 2010, S. 133f). Im fachwissenschaftlichen und fachpraktischen Diskurs finden sich für die Tätigkeit der Begleitung des Schulbesuchs verschiedene synonym verwendete Bezeichnungen wie bspw. Schulassistenz, Schulbegleitung, Integrationshilfe, Inklusionshilfe oder Inklusionsassistenz (vgl. z. B. Dworschak, 2010; Meyer, 2017). Im vorliegenden Beitrag wird der Begriff Schulassistenz verwendet, da er sowohl die Unterstützung von Bildungsprozessen impliziert als auch den Selbstbestimmungsanspruch des Leistungsempfängers berücksichtigt. Der Begriff Assistenz vermeidet dabei eine Bevormundung des Leistungsempfängers und wendet sich gegen eine fremdbestimmte Behindertenhilfe, die u. U. durch Termini wie Schulhelfer suggeriert wird.

\subsection{Schulassistenz-Bewilligungszahlen}

Schulassistenz ist in den letzten Jahren zu einem wichtigen Bestandteil des Schulalltags geworden. Hierauf deuten vor allem die wachsenden Bewilligungszahlen hin. Wenngleich die Inanspruchnahme von Schulassistenz in der amtlichen Statistik nicht gesondert erfasst wird, lässt sich bereits für den Zeitraum zwischen 2010 - etwa 26.000 Fälle und 2013 - etwa 46.000 Fälle - eine deutliche Zunahme schulbezogener Eingliederungshilfen ausmachen (vgl. Meyer, 2017). Erhebungen zur Entwicklung der Fallzahlen an Förderschulen (vgl. Kißgen et al., 2013; Beck et al., 2010) und allgemeinen Schulen (vgl. Dworschak, 2014 a) unterstützen die Annahme einer stetigen Zunahme schulbezogener Eingliederungshilfen.

Heinrich und Lübeck (2013) führen den wachsenden Einsatz von Schulassistent/innen vor allem auf einen Mangel an Lehrer/innen für Sonderpädagogik zurück (vgl. auch Klemm, 2012; Benus \& Weidemann, 2016). Zahlreiche Schulassistent/innen stehen aus diesem Grund nicht nur vor der Aufgabe, individuelle Hilfen zu einer angemessenen Schulbildung zu leisten, sondern auch vor der Herausforderung, Mängel in der Personalversorgung auszugleichen. Für Fachkräfte mit jahrelanger Berufserfahrung ebenso wie für junge Erwachsene im freiwilligen sozialen Jahr ist dies eine höchst anspruchsvolle, herausfordernde und bisweilen gar überfordernde Aufgabe (Heinrich \& Lübeck, 2013) mit unbekanntem Einfluss auf die kindliche Entwicklung.

\subsection{Schulassistenz - Forschungs- überblick und -desiderata}

Die empirische Forschung zum Thema Schulassistenz steht in Deutschland noch am Anfang. Erst im Zuge der UN-Behindertenrechtskon- 
vention und der zuletzt stark wachsenden Bewilligungszahlen gewinnt Schulassistenz als Forschungsgegenstand allmählich an Interesse (vgl. Lübeck \& Demmer, 2017). Im Mittelpunkt der wenigen bisherigen Forschungsarbeiten steht vor allem die Beschaffung grundlegender Informationen zu Merkmalen und Strukturen von Schulassistenz. Die zumeist quantitativen Arbeiten werden durch wenige qualitative Studien ergänzt (vgl. Dworschak 2014b; Schmidt, 2017). Zwei aktuelle qualitative Studien haben Böing und Köpfer (2017) sowie Lindmeier und Ehrenberg (2017) vorgestellt. Im Projekt „Schulassistenz aus Schüler/innenperspektive" wurden u. a. Informationen dazu erhoben, wie Lernende die ihnen zugewiesene Assistenz erlebten, wie sie sich in der Assistenzsituation verhielten und wie sie lernten. Die Zwischenergebnisse ermöglichen interessante Eindrücke zu den Zielen von Schulassistenz im Unterrichtsalltag: Während Unterstützungsleistungen zur Förderung der persönlichen Entwicklung lediglich eine nachgeordnete Rolle spielten, lag der Schwerpunkt der Assistenz in kompensatorischen Handlungen bspw. zum Ausgleich von Leistungs- oder Verhaltensabweichungen (Böing \& Köpfer, 2017). In der Studie von Lindmeier und Ehrenberg (2017) lag der Fokus hingegen auf der Perspektive der Mitschüler/innen sowie auf Schulassistenz und ihren Auswirkungen. In Gruppendiskussionen wurden Einschätzungen zur sozialen Partizipation schulbegleiteter Kinder und zum Handeln von Schulassistent/innen erhoben. Es zeigte sich, dass die Mitschüler/innen Schulassistenz durch ihre Einzelfallorientierung als Ungleichbehandlung wahrnahmen. Aus Sicht der Mitschüler/innen erzeugte Schulassistenz zudem eine Differenz zwischen der Schülerschaft und beeinflusste die Gestaltung von Peerinteraktionen.

Zur Wirkung von Schulassistenz auf die Entwicklung begleiteter Schüler/innen existieren bislang nur wenige Erkenntnisse (vgl. Lübeck
\& Demmer, 2017). Bei den vorhandenen Studien handelt es sich um deskriptiv ausgerichtete Untersuchungen aufbauend auf Fragebogenerhebungen mit prozessbeteiligten Personen größtenteils im Querschnitt.

Eine erste Annäherung an das Thema hat Wohlgemuth (2009) unternommen. In einer Querschnittsbefragung wurde die Wirkung von Schulassistenz auf begleitete Schüler/innen aus Sicht der Schulassistent/innen erhoben. Der Einfluss von Schulassistenz auf die soziale und schulische Integration wurde im Mittel als positiv beurteilt, ebenso wie die Einschätzung hinsichtlich der leistungsbezogenen Integration. Die befragten Schulassistent/innen berichteten diesbezüglich im Mittel von einer Verbesserung der Situation ${ }^{1}$.

Die Forschergruppe um Dworschak (Beck et al., 2010; Dworschak, 2012 a; Dworschak, 2012 b) hat in drei Untersuchungen Grundlagenforschung zur Schulassistenz geleistet. Im Rahmen einer Querschnittsbefragung wurde die Wirkung von Schulassistenz anhand der Faktoren Integration und Gesamtentwicklung operationalisiert. Schulassistent/innen und Klassenlehrkräfte bewerteten den Einfluss von Schulassistenz auf die Integration in die Klassengemeinschaft i.d.R. als positiv. Die Gesamtsituation der begleiteten Schüler/innen schätzten $90 \%$ der Schulassistent/innen und Klassenlehrkräfte als verbessert ein. Bei einer Replikationsstudie zur Schulassistenz bei Schüler/innen mit Unterstützungsbedarf im Förderschwerpunkt Geistige Entwicklung (Dworschak, 2012 a) sahen ebenfalls 90\% der Schulassistent/innen und $79 \%$ der Klassenlehrkräfte eine positive Wirkung von Schulassistenz auf die Integration in die Klassengemeinschaft. In einer weiteren querschnittlichen Studie (Dworschak, 2012 b) gaben 95\% der Befragten an, dass sich Schulassistenz positiv auf die Integration in der Klassengemeinschaft auswirke. 
In der Studie von Zauner und Zwosta (2014) wurden 50 Klassenlehrkräfte, 54 Schulassistent/innen sowie jeweils 36 Eltern und Schüler/innen retrospektiv zu den Effekten von Schulassistenz befragt. Ein Großteil der befragten Personen schätzte die Wirkung von Schulassistenz als positiv ein - insbesondere die Gesamtentwicklung, die Entwicklung des Sicherheitsgefühls sowie der Konzentrationsfähigkeit. Uneinheitliche Einschätzungen fanden sich bezüglich der Freude am Schulbesuch: Während Schüler/innen und Eltern die Freude der Kinder am Schulbesuch mit 61 \% bzw. $67 \%$ als positiv verbessert einschätzten, teilten unter den Lehrer/innen nur $46 \%$ diese Auffassung. Kritisch beurteilt wurden daneben stigmatisierende und belastende Effekte von Schulassistenz. So beschreiben jeweils etwa $30 \%$ der befragten Lehrer/innen und Schüler/innen zumindest eingeschränkt stigmatisierende oder belastende Effekte.

Eine aktuelle Studie zur Schulassistenz haben Markowetz und Jerosenko (2016) mit dem Modellprojekt Integrationshelfer in der inklusiven Schule vorgestellt. Über den Zeitraum von vier Jahren wurden sowohl querschnittliche als auch längsschnittliche Daten erhoben. Nach Einschätzung der Lehrpersonen und Schulassistent/innen zeigten die sozial-emotionalen Kompetenzen begleiteter Schüler/innen eine fast ausnahmslos positive Entwicklung über die Zeit. Anders fiel die Einschätzung bezüglich der sozialen Integration aus: Schüler/innen mit Schulassistenz wie auch Mitschüler/innen kamen bezüglich der sozialen Integration übereinstimmend zu einer leicht negativen Einschätzung.

Im Vergleich zu den für Deutschland verfügbaren Daten ist der internationale Forschungsstand zur Wirkung von Schulassistenz umfangreicher. Bezüglich der Entwicklung des Sozialverhaltens von Schüler/innen mit Schulassistenz konnten Malmgren und CaustonTheoharis (2006) einen negativen Einfluss von Schulassistenz auf das Interaktionsverhalten nachweisen. Auch Symes und Humphrey (2012) stellten einen negativen Einfluss auf Selbstständigkeit, Sozialkontakt und Hilfsbereitschaft in Lernsituationen fest. Bzgl. der Lernentwicklung bei Schulassistenz zeigte sich, dass - obgleich Schulassistenz das Lernen von Kindern positiv unterstützen kann, wenn Maßnahmen zielgerichtet und mit hoher Qualität erfolgen die bloße Anwesenheit von Schulassistenz zunächst keinen positiven Effekt hat (vgl. Farrell, Alborz, Howes \& Pearson, 2010). Webster et al. (2010) stellten sogar einen negativen Zusammenhang zwischen Lernzuwachs und Schulassistenz fest und kommen zu dem Schluss, dass der Lernzuwachs begleiteter Schüler/innen umso geringer ausfällt, je umfangreicher die Schulassistenz ist. Die ungünstige Wirkung von Schulassistenz auf Lernzuwachs, Lernund Sozialverhalten führen Sharma und Salend (2016) u. a. auf den verbreiteten Einsatz gering qualifizierter und unbeaufsichtigter Schulassistent/innen zurück.

Schulassistenz - dies lässt sich zusammenfassend festhalten - ist ein bislang nur wenig erschlossenes Forschungsgebiet. Insbesondere die Entwicklung von Schüler/innen mit Schulassistenz wird in deutschsprachigen Studien nur beiläufig behandelt und allenfalls deskriptiv ausgewertet. Der vorliegende Beitrag setzt an diesem Forschungsdesiderat an.

\subsection{Lern- und Sozialverhalten als Bedingungsfaktoren für einen erfolgreichen Schulbesuch}

Im Zusammenhang mit Schulassistenz ist die Betrachtung der personenbezogenen Faktoren von Schulerfolg von besonderem Interesse. $\mathrm{Da}$ Schüler/innen und Schulassistent/innen in der Regel über längere Zeit intensiv zusammenarbeiten, kann Schulassistenz eine besondere Relevanz für die Entwicklung von Eigenschaften für einen erfolgreichen Schulbesuch unterstellt werden. 
$\mathrm{Zu}$ den Bedingungsfaktoren von Schulerfolg liegen unterschiedliche Modelle und Studien vor, welche übereinstimmend die Bedeutung der Lehr- und Lernprozesse betonen (vgl. Carroll, 1963; Walberg, 1984; Wang, Haertel \& Walberg, 1993; Hattie, 2008). Nach neueren Erkenntnissen sind es vor allem die Eigenschaften der Lernenden, die über Schul- und Lernerfolg bestimmen (vgl. Wang et al. 1993; Hattie, 2008). In Hatties Visible Learning Research $(2008 ; 2015)$ konnte diesbezüglich gezeigt werden, dass personenbezogene Faktoren wie Vorwissen, Begabung, Lernmotivation, Ziele oder bevorzugte Lernaktivitäten etwa 50 \% der Unterschiede im Lernerfolg erklären.

Im Zusammenhang mit Schulassistenz rücken vor allem verhaltensbezogene Faktoren von Schulerfolg wie Lern- und Sozialverhalten in den Vordergrund, da diese veränderlich sind und im Unterschied zu Begabung oder Intelligenz in der täglichen Zusammenarbeit adressiert werden können.

Studien weisen darauf hin, dass sowohl das Lernverhalten - im Sinne unterrichtsbezogener Fähigkeiten und Fertigkeiten wie etwa dem Folgen von Anweisungen, der Arbeitsplatzorganisation, zielorientiertem Arbeiten und genauem Zuhören (McClelland, Morrison \& Holmes, 2000) - als auch das Sozialverhalten Schulerfolg erheblich beeinflussen. Das Unterrichtsverhalten weist typischerweise eine hohe zeitliche Stabilität auf und wirkt somit langfristig erschwerend oder unterstützend auf Schulerfolg (Romano, Babchishin, Pagani \& Kohen, 2010). Als besondere Risikofaktoren für einen erfolgreichen Schulbesuch gelten externalisierende Verhaltensweisen wie Impulsivität (Moilanen, Shaw \& Maxwell, 2010), Substanzmissbrauch (Ansary \& Luthar, 2009), Hyperaktivität, Aufmerksamkeitsprobleme, antisoziales und kriminelles Verhalten (Hinshaw, 1992) u. a., da sie Lehrer-Kind-Konflikte fördern und die Gefahr sozialer Ausgrenzung bergen (Newcomb, Bukowski \& Pattee, 1993).
Hierdurch kann die Teilhabe am Lernen gefährdet und der Schulerfolg negativ beeinflusst werden (Ladd, Birch \& Buhs, 1999). Internalisierenden Verhaltensproblemen wie z. B. Ängstlichkeit wird hingegen ein geringerer Einfluss auf Schulerfolg zugeschrieben (vgl. Ansary \& Luthar, 2009).

In Anbetracht der besonderen Bedeutung des Lern- und Sozialverhaltens für einen erfolgreichen Schulbesuch und vor dem Hintergrund des Anliegens von Schulassistenz, Unterstützung beim Schulbesuch zu leisten, widmet sich die vorliegende Arbeit der Entwicklung des Lern- und Sozialverhaltens bei Schüler/innen mit Schulassistenz.

Der Beitrag geht den folgenden Forschungsfragen nach:

Wie entwickeln sich das Lern- und Sozialverhalten von Schüler/innen mit Schulassistenz über den Zeitraum eines Schuljahres hinweg? Insbesondere Wie entwickeln sich das Lernverhalten und seine Faktoren? sowie Wie entwickeln sich das Sozialverhalten und seine jeweiligen Faktoren?

Bei der Beantwortung dieser Fragen werden auch Subgruppen von Schüler/innen betrachtet, z. B. werden Unterschiede zwischen Mädchen und Jungen sowie zwischen Schüler/innen der allgemeinen und der Förderschule untersucht.

\section{Methodologie}

Bei der vorliegenden Untersuchung handelt es sich um eine Längsschnittbefragung von Klassenlehrkräften an allgemeinen und Förderschulen. Die Untersuchungsteilnehmer/innen wurden zweimal - zu Beginn und gegen Ende eines Schuljahres - im Abstand von sieben Monaten zum Lern- und Sozialverhalten schulbegleiteter Schüler/innen befragt. Als Erhe- 
bungsinstrument kam die Lehrereinschätzliste für Sozial- und Lernverhalten (LSL) (Petermann \& Petermann, 2013) zum Einsatz.

\subsection{Wahl des Erhebungsinstruments}

Die LSL zeichnet sich aus durch eine hohe Reliabilität $(\alpha>0.8)$ und eine große Normierungsstichprobe mit Normwerten für Schüler/innen im Alter von 6 bis 19 Jahren. Aufgrund ihrer Eignung zur Verlaufsbeurteilung bietet die LSL auch die Möglichkeit zum Einsatz in Längsschnittuntersuchungen. Für Lehrpersonen ist das Verfahren in wenigen Minuten durchführbar. Mit der LSL werden das Lernverhalten mit insgesamt vier Subskalen und das Sozialverhalten mit sechs Subskalen erfasst.

\subsection{Wahl der Erhebungszeitpunkte}

\subsubsection{Entwicklungspsychologische Perspektive}

Die Lehrer/innenbefragung wurde zu zwei Erhebungszeitpunkten im Herbst $2015\left(\mathrm{t}_{1}\right)$ und im Frühjahr $2016\left(t_{2}\right)$ durchgeführt. Hierbei wurden Informationen über Schüler/innen im Alter zwischen 6 und 19 Jahren erhoben. Während dieser Entwicklungsphase weisen Kinder und Jugendliche eine leicht verringerte Stabilität sozial-emotionaler Persönlichkeitsmerkmale auf (Roberts \& DelVecchio, 2000). Sowohl altersgemäß wie auch mit Blick auf schulassistenzbedingte Umweltveränderungen und Resilienz (Asendorpf \& Neyer, 2012) befanden sich die Untersuchungsteilnehmer/innen in einer sensiblen Phase der Persönlichkeitsentwicklung. Mit zwei Erhebungszeitpunkten im Abstand von sieben Monaten trägt der Modus der Datenerhebung den beschriebenen Bedingungen Rechnung und bietet ausreichend Raum für Entwicklung und Veränderung der Schüler/innenpersönlichkeit im Rahmen von Schulassistenz.

\subsubsection{Organisatorische Perspektive}

Um eine möglichst geringe Ausfallrate im Sinne der Minimierung der statistischen Mortalität zu gewährleisten, wurden bei der Festlegung der Erhebungszeitpunkte sowohl das Anstellungsverhältnis der Schulassistent/innen als auch die zeitliche Begrenzung von Eingliederungshilfe sowie schulorganisatorische Bedingungen und Übergänge berücksichtigt.

Schulassistent/innen werden zumeist in einem befristeten Arbeitsverhältnis beschäftigt (vgl. Heinrich \& Lübeck, 2013). Bei Personen, die im Rahmen eines Freiwilligendienstes als Schulassistenz tätig sind, umfasst die Dienstzeit häufig ein Schuljahr und endet mit Beginn der Sommerferien. Neben dem Arbeitsverhältnis legen auch das Hilfeplanverfahren bzw. der Gesamtplan eine zeitliche Begrenzung der Datenerhebung nahe. Die gewährten Leistungen sind demzufolge nicht auf Dauer angelegt, sondern erfolgen mit dem Ziel einer sukzessiven Reduktion der Unterstützung bis zu ihrer vollständigen Aufhebung (vgl. Henn et al., 2014). Die Beendigung der Eingliederungshilfe als Ausfallursache kann daher nicht vollständig ausgeschlossen werden - ihr wird jedoch durch die Wahl einer überschaubaren Zeitspanne von sieben Monaten zwischen den Erhebungszeitpunkten entgegengewirkt. Zudem werden durch den gewählten Erhebungszeitraum auch schulorganisatorische Einflüsse wie Klassenleitungswechsel, Schulwechsel oder das Ende der Pflichtschulzeit als Ausfallursachen minimiert.

\subsection{Beteiligte Personen}

Insgesamt wurden 71 Dienste für Schulassistenz kontaktiert. Diese setzten sich zusammen aus 24 privaten Anbietern und 47 Einrichtungen der Freien Wohlfahrtspflege. Das Forschungsvorhaben wurde allen Interessenten telefonisch, zum Teil persönlich vor Ort oder in Arbeitskreisen vorgestellt sowie in schriftlicher Form zur Verfügung gestellt. An der Datenerhebung 
haben schließlich neun Dienste für Schulassistenz aus NRW mitgewirkt. Diese Kooperation war Grundlage für die Kontaktaufnahme zu Schulen, Eltern und Schulassistent/innen. Unter den teilnehmenden Schulen waren sowohl Grundschulen als auch Förderschulen und weiterführende Schulen. An der Erstbefragung $\left(\mathrm{t}_{1}\right)$ nahmen insgesamt 82 Klassenlehrkräfte teil. 70 Lehrpersonen waren bereit, ihre Einschätzung am Schuljahresende $\left(\mathrm{t}_{2}\right)$ erneut abzugeben. Dies entspricht einer Ausfallrate von 14,6\%.

\subsection{Datenanalyse}

Im vorliegenden Beitrag werden längsschnittliche Informationen über das Lern- und Sozialverhalten von 65 Schüler/innen mit Schulassistenz sowie grundlegende demografische Daten analysiert. Es erfolgen Analysen zu Unterschieden in den Entwicklungsverläufen des Lern- und Sozialverhaltens in der Gesamtstichprobe sowie in verschiedenen Subgruppen.
Hauptanliegen der Datenanalyse ist die Prüfung von Unterschieden zwischen den Erhebungszeitpunkten $t_{1}$ und $t_{2}$. Als statistisches Verfahren bei kleinen Stichproben wird der Wilcoxon-Test eingesetzt. Das Testverfahren prüft, ob sich zwei abhängige Stichproben in ihrer zentralen Tendenz unterscheiden. Hierfür werden sowohl die Richtung des Unterschieds als auch die Größe des Unterschieds paariger Messungen genutzt (Bortz \& Lienert, 2008). Als parametrisches Pendant zum Wilcoxon-Test kommt daneben der $t$-Test zum Einsatz, sofern die Voraussetzungen erfüllt sind.

\section{Ergebnisse}

\subsection{Deskriptive Statistik}

Ergänzend zum Lern- und Sozialverhalten wurden verschiedene soziodemografische Daten der Schüler/innenpopulation erfasst, welche Tabelle 1 zu entnehmen sind.

Tab. 1 Soziodemografische Daten der Schüler/innenpopulation: Alter, Geschlecht, Sonderpädagogischer Unterstützungsbedarf, Schulform, Bewilligungsstatus

\begin{tabular}{|c|c|c|c|}
\hline & weiblich & männlich & gesamt \\
\hline$N(\%)$ & $17(26.2)$ & $48(73.8)$ & $65(100)$ \\
\hline Alter in Jahren: $M(S D)$ & $9.18(2.01)$ & $10.85(2.99)$ & $10.42(2.85)$ \\
\hline Sonderpäd. Unterstützungsbedarf: N (\%) & $14(82.3)$ & $39(81.3)$ & $53(81.5)$ \\
\hline $\begin{array}{l}\text { Schulform: } N(\%) \\
\text { Grundschule } \\
\text { Förderschule } \\
\text { - Hauptschule } \\
\text { Gesamtschule } \\
\text { - Sekundarschule } \\
\text { - Realschule } \\
\text { Gymnasium } \\
\text { Gine andere Schulform }\end{array}$ & $\begin{array}{c}8(47.1) \\
2(11.8) \\
4(23.5) \\
1(5.9) \\
0(0) \\
1(5.9) \\
0(0) \\
1(5.9)\end{array}$ & $\begin{array}{c}18(37.5) \\
9(18.6) \\
6(12.5) \\
8(16.7) \\
3(6.3) \\
1(2.1) \\
1(2.1) \\
1(2.1)\end{array}$ & $\begin{array}{c}26(40.0) \\
11(16.9) \\
10(15.4) \\
9(13.8) \\
3(4.6) \\
2(3.1) \\
1(1.5) \\
2(3.1)\end{array}$ \\
\hline $\begin{array}{l}\text { Bewilligungsstatus: N (\%) } \\
\text { Erstbewilligung } \\
\text { Folgebewilligung }\end{array}$ & $\begin{array}{c}3(17.6) \\
14(82.4)\end{array}$ & $\begin{array}{l}11(22.9) \\
36(75.0)\end{array}$ & $\begin{array}{l}14(21.5) \\
50(76.9)\end{array}$ \\
\hline
\end{tabular}




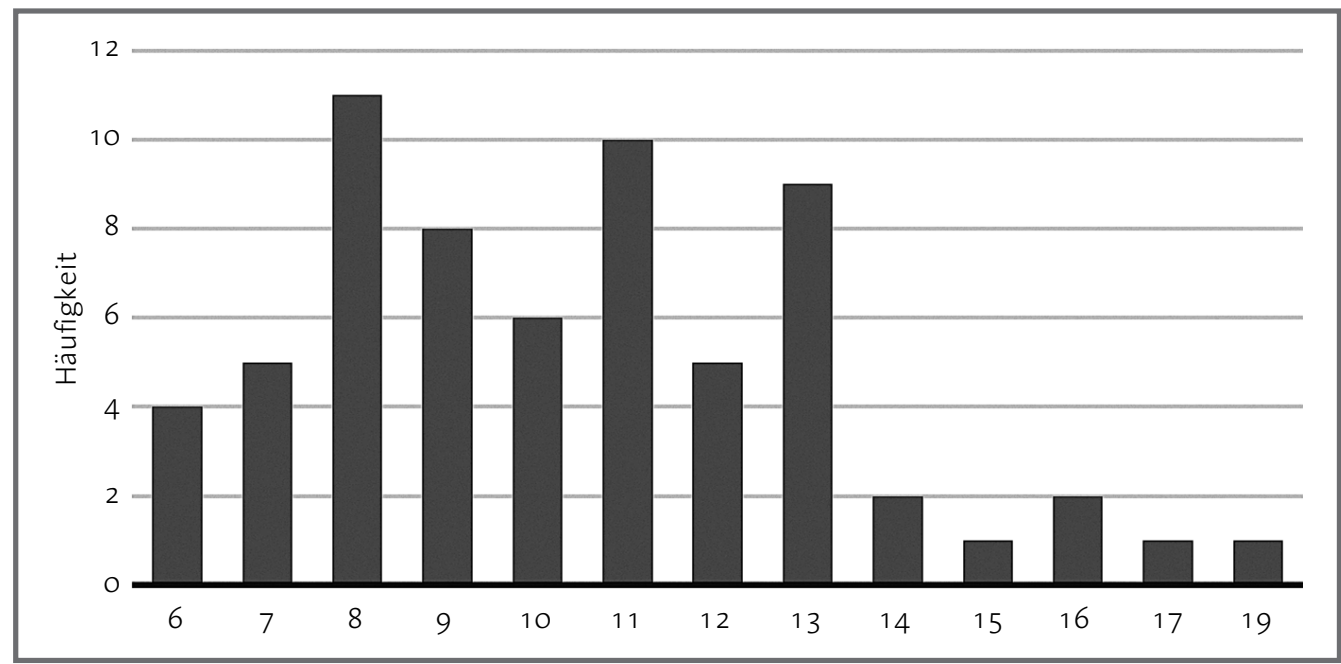

Abb. 1 Lebensalter der Schüler/innenpopulation in Jahren zu Zeitpunkt $t_{1}$

Das Durchschnittsalter der Schüler/innenpopulation lag zum Zeitpunkt der Erstbefragung bei 10.42 Jahren $(S D=2.85)$. Die jüngsten Schüler/innen waren 6 Jahre alt, der älteste Schüler 19 Jahre. Die Altersspanne betrug 13 Jahre bei einem Rückgang der Fallzahlen mit zunehmendem Alter (vgl. Abb. 1). Das männliche Geschlecht (73.8\%) überwog deutlich das weibliche (26.2\%). Mehr als drei Viertel (81.5\%) der Schüler/innen wiesen einen sonderpädagogischen Unterstützungsbedarf auf, wobei die Anteile zwischen den Geschlechtern hier gleich verteilt waren.

Unter den Schulformen war die Grundschule (40.0\%) am häufigsten vertreten. Jeweils etwa 15 Prozent besuchten eine Förderschule (16.9\%), Hauptschule (15.4\%) oder Gesamtschule (13.8\%). Die weiteren Schulformen waren im einstelligen Prozentbereich angesiedelt. Bei Betrachtung der Daten wird ein Rückgang der Fallzahlen im gegliederten Schulsystem der Sekundarstufe von der Hauptschule $(N=10)$ bis zum Gymnasium $(N=1)$ deutlich.

Für vertiefende Einblicke in die Merkmale der vorliegenden Stichprobe bietet sich eine Aufgliederung in Subgruppen an (s. Tab. 2). Es zeigen sich ungleiche Verteilungen bezüglich der Lernorte und Bewilligungen. 54 Schüler/innen $(83.1 \%)$ besuchten eine allgemeine Schule, 11 (16.9\%) eine Förderschule. Bei den Bewilligungen zeigte sich ein starkes Übergewicht an Folgebewilligungen bei insgesamt 50 Schüler/innen (83.1\%) gegenüber einem geringen Anteil Erstbewilligungen - bei 11 Schüler/innen (16.9\%). Die Aufgliederung in Subgruppen erwies sich auch bezüglich des Zusammenhangs zwischen Bewilligungsstatus und Alter als aufschlussreich. Während sich bei den 6- bis 9-jährigen Schüler/innen Erstbewilligungen (42.9\%) und Folgebewilligungen (57.1\%) etwa die Waage hielten, überwog bei den 10- bis 19-jährigen Schüler/innen bei Weitem der Anteil der Folgebewilligungen (91.8\%). In der späten Kindheit und Jugend scheint Schulassistenz vor allem eine bewährte und fortlaufende Maßnahme zu sein, während in der frühen Kindheit eher die Erstbewilligung von Schulassistenz erfolgt (vgl. Abb. 2). Die Kontingenzanalyse der Variablen Alter und Bewilligungsstatus (s. Tab. 2) verdeutlicht dies. Hierbei verweist die Prozentsatzdifferenz von 37.5 Prozent im Bereich Erstbewilligung auf einen Zusammenhang zwischen Alter und Einrichtung einer Schulassistenz. 


\begin{tabular}{|c|c|c|c|c|c|}
\hline مَ & 은 & 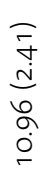 & $\begin{array}{l}\widetilde{0} \\
\infty \\
\infty \\
\dot{q} \\
\dot{q}\end{array}$ & & 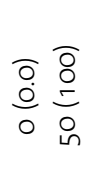 \\
\hline 岕 & $\underset{\leftarrow}{\ddagger}$ & 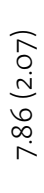 & $\begin{array}{l}\tilde{m} \\
\dot{\theta} \\
\text { a } \\
a\end{array}$ & & 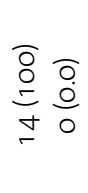 \\
\hline 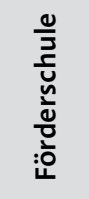 & $\mp$ & 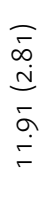 & $\begin{array}{l}5 \\
8 \\
5 \\
5\end{array}$ & & $\begin{array}{l}\hat{\sigma} \\
\dot{\sigma} \\
\dot{\sigma} \\
-\quad \\
\circ\end{array}$ \\
\hline 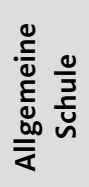 & L & 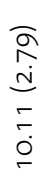 & $\begin{array}{l}\underset{+}{N} \\
\stackrel{n}{n} \\
\end{array}$ & & 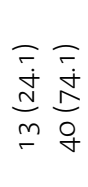 \\
\hline $\begin{array}{l}0 \\
\frac{0}{2} \\
\frac{1}{0} \\
\sigma \\
1 \\
1 \\
0 \\
0\end{array}$ & $\hat{m}$ & 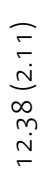 & 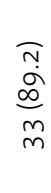 & & 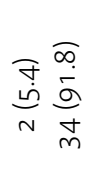 \\
\hline $\begin{array}{l}\frac{0}{2} \\
\frac{2}{\pi} \\
a \\
1 \\
0\end{array}$ & $\stackrel{\infty}{N}$ & $\begin{array}{l}\widehat{N} \\
0 \\
\\
N \\
\infty \\
N \\
N\end{array}$ & $\begin{array}{l}\mathcal{F} \\
\stackrel{N}{N} \\
\text { O }\end{array}$ & & 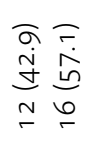 \\
\hline & $z$ & 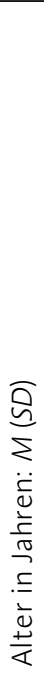 & 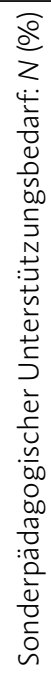 & 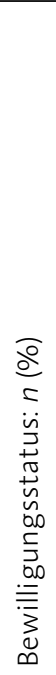 & 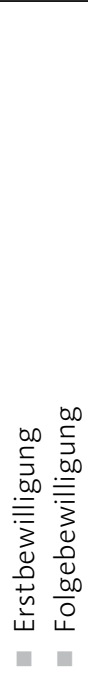 \\
\hline
\end{tabular}

Tab. 3 Kreuztabelle Geschlecht $\times$ Alter

\begin{tabular}{|c|c|c|}
\hline & Gesc & lecht \\
\hline & weiblich & männlich \\
\hline $\begin{array}{l}6-9 \text { Jahre } \\
10-19 \text { Jahre }\end{array}$ & $\begin{array}{l}64.7 \% \\
35.3 \%\end{array}$ & $\begin{array}{l}35.4 \% \\
64.4 \%\end{array}$ \\
\hline$n$ & 17 & 48 \\
\hline
\end{tabular}

Hinweise auf Zusammenhänge fanden sich auch bei der Betrachtung der Variablen Alter und Geschlecht (s. Tab. 3). Die Prozentsatzdifferenzen weisen darauf hin, dass Mädchen Schulassistenz eher während der frühen und mittleren Kindheit erhalten, während bei Jungen die späte Kindheit und Adoleszenz überwiegen. Generell werden Jungen, verglichen mit Mädchen, deutlich häufiger mit Schulassistenz versorgt.

\subsection{Lern- und Sozialverhalten im Vergleich zur Eichstichprobe}

Die Ergebnisdarstellung beginnt mit einem Vergleich der vorliegenden Daten zu den Referenzdaten der Eichstichprobe von Petermann und Petermann (2003). Der Datenabgleich erfolgt auf Grundlage der Rohwerte beider Datensätze und vermittelt einen ersten Eindruck zur Entwicklung des Lern- und Sozialverhaltens bei Schüler/innen mit Schulassistenz (s. Abb. 3).

Die Schüler/innen mit Schulassistenz schnitten im Vergleich zur Eichstichprobe in allen Subskalen deutlich schwächer ab. Im Mittel erreichten sie mit $M=7.58(S D=3.60) 2.95$ Rohwertpunkte weniger als der Durchschnitt der Eichstichprobe $(M=10.53)$. Am deutlichsten war dieser Unterschied im Aussagebereich Lernverhalten: In der Subskala Selbstständigkeit erreichten die Schüler/innen mit Schulassistenz bei Rohwerten von $M=4.98(S D=3.23)$ 


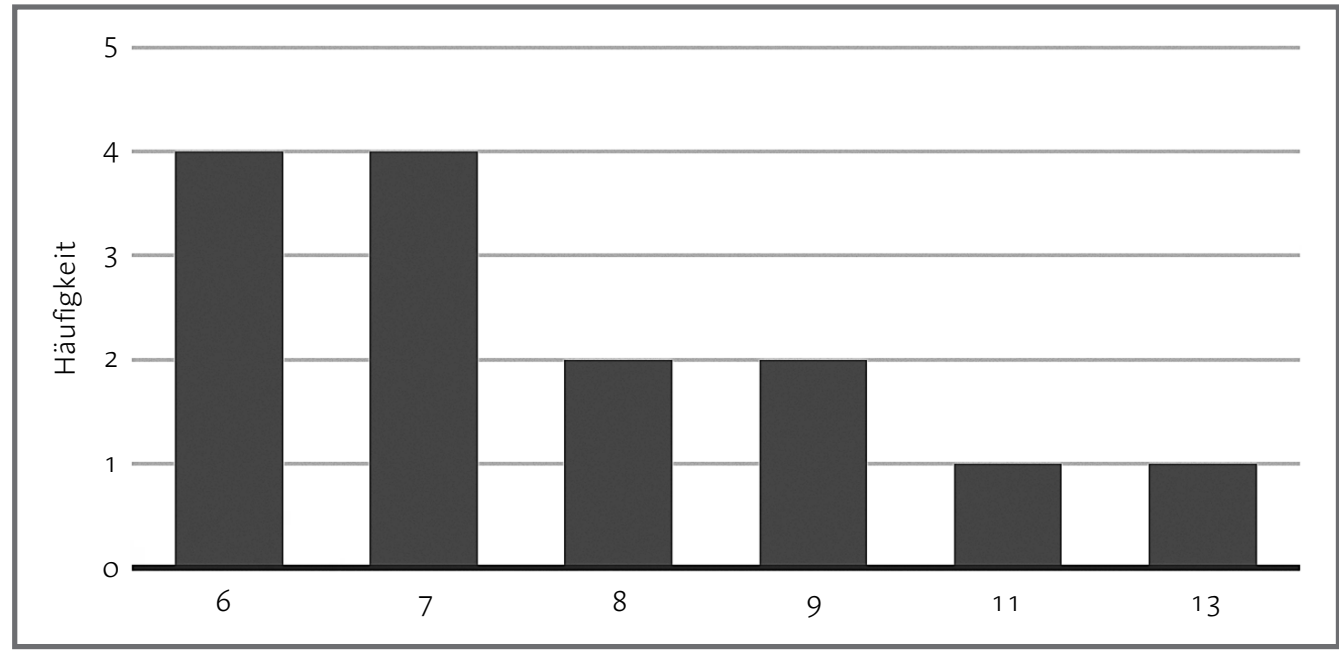

Abb. 2 Lebensalter der Schüler/innen mit Erstbewilligung in Jahren zu Zeitpunkt $t_{1}$

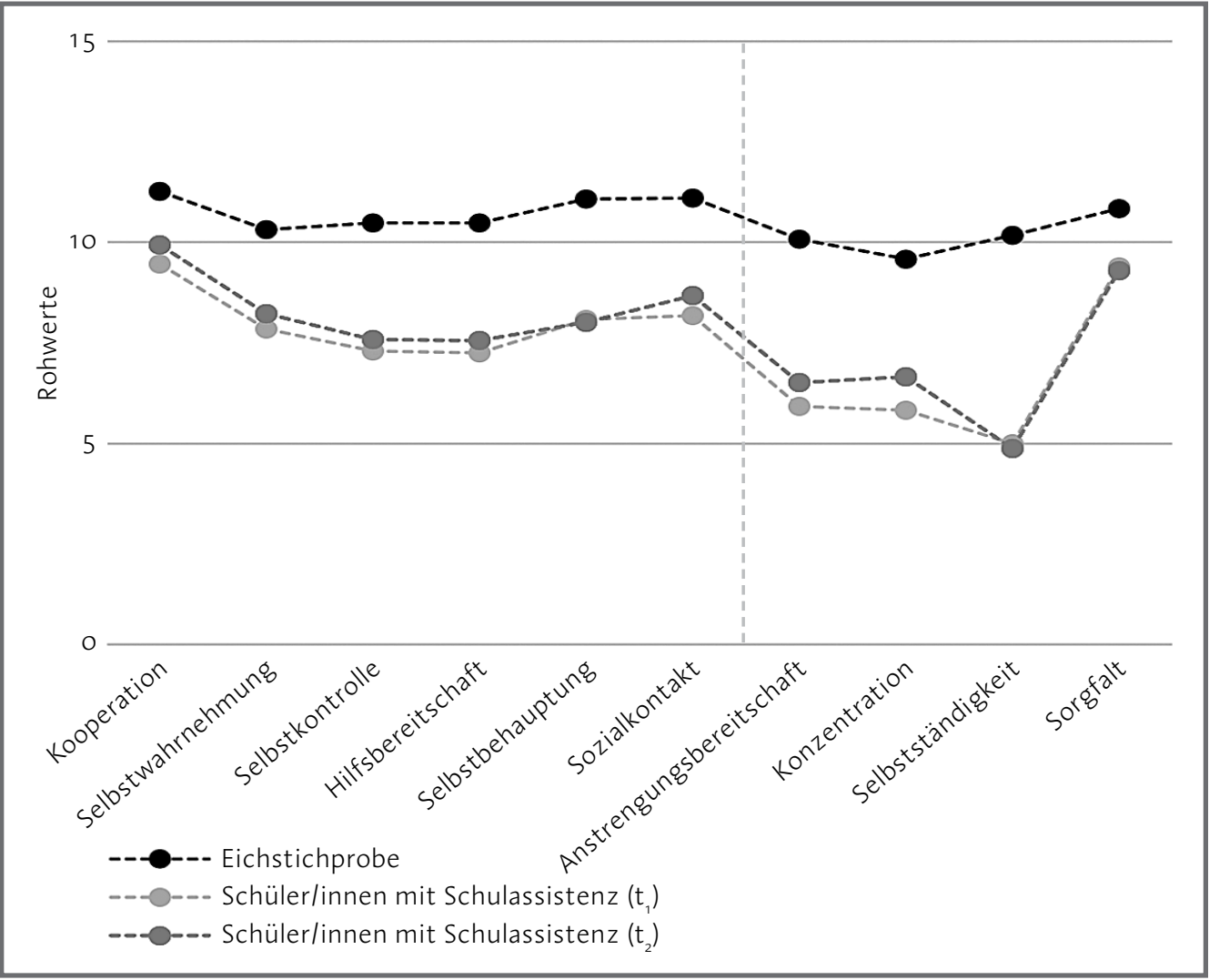

Abb. 3 Subskalen der Eichstichprobe und der Schüler/innen mit Schulassistenz zu den Zeitpunkten $t_{1}$ und $t_{2}(R W$ im Mittel, $N=65)$ 
zu Zeitpunkt $\mathrm{t}_{1}$ und $M=4.86(S D=3.26) \mathrm{zu}$ Zeitpunkt $t_{2}$ im Vergleich zur Eichstichprobe $(M=10.17)$ in beiden Erhebungen weniger als die Hälfte der Punkte. Bemerkenswert sind auch die Unterschiede zu Erhebungszeitpunkt $t_{1}$ in den Subskalen Anstrengungsbereitschaft und Konzentration. Mit Rohwerten von $M=5.91(S D=3.45)$ in der Subskala Anstrengungsbereitschaft und $M=5.81(S D=3.53) \mathrm{im}$ Bereich Konzentration wiesen die Schüler/innen mit Schulassistenz in beiden Subskalen eine deutlich negative Abweichung von der Eichstichprobe auf. In den Subskalen Kooperation und Sorgfalt waren die Unterschiede zwischen Eichstichprobe und Schüler/innen mit Schulassistenz am geringsten ausgeprägt.

\subsection{Analyse von Entwicklungsverläufen}

Zur Prüfung von Unterschieden zwischen den Erhebungszeitpunkten $t_{1}$ und $t_{2}$ wurden Tests auf Lageunterschied mit den Faktoren Lehrer/inneneinschätzung (T-Werte) und Zeit $\left(t_{1}, t_{2}\right)$ gerechnet. Wenn die Normalverteilungsvoraussetzung erfüllt war und eine ausreichend große Stichprobe vorlag, wurde der $t$-Test für verbundene Stichproben eingesetzt. War die Voraussetzung für eine parametrische Testung nicht erfüllt, kam der Wilcoxon-Test zum Einsatz. Als Effektstärkemaß wurde Pearson's Korrelationskoeffizient $(r)$ verwendet. Die Interpretation erfolgte nach den Konventionen von Cohen (1988). In die Analyse wurden neben der Gesamtstichprobe folgende Subgruppen aufgenommen: männlich, weiblich, Erstbewilligung, Folgebewilligung, Allgemeine Schule, Förderschule, 6 bis 9 Jahre, 10 bis 19 Jahre. Die Tabellen 4 und 5 bieten einen Überblick ausgewählter Analyseergebnisse.

\subsubsection{Aussagebereich Sozialverhalten}

Zur Prüfung von Unterschieden zwischen Erstund Folgebefragung wurden die T-Werte der LSL-Subskalen addiert und hieraus Mittelwerte bestimmt. Für die Analyse wurde die gesamte Stichprobe mit $N=65$ Fällen herangezogen.

Im Aussagebereich Sozialverhalten kam es während des Untersuchungszeitraums zu keiner signifikanten Veränderung $(t=-1.010, p=.361$,

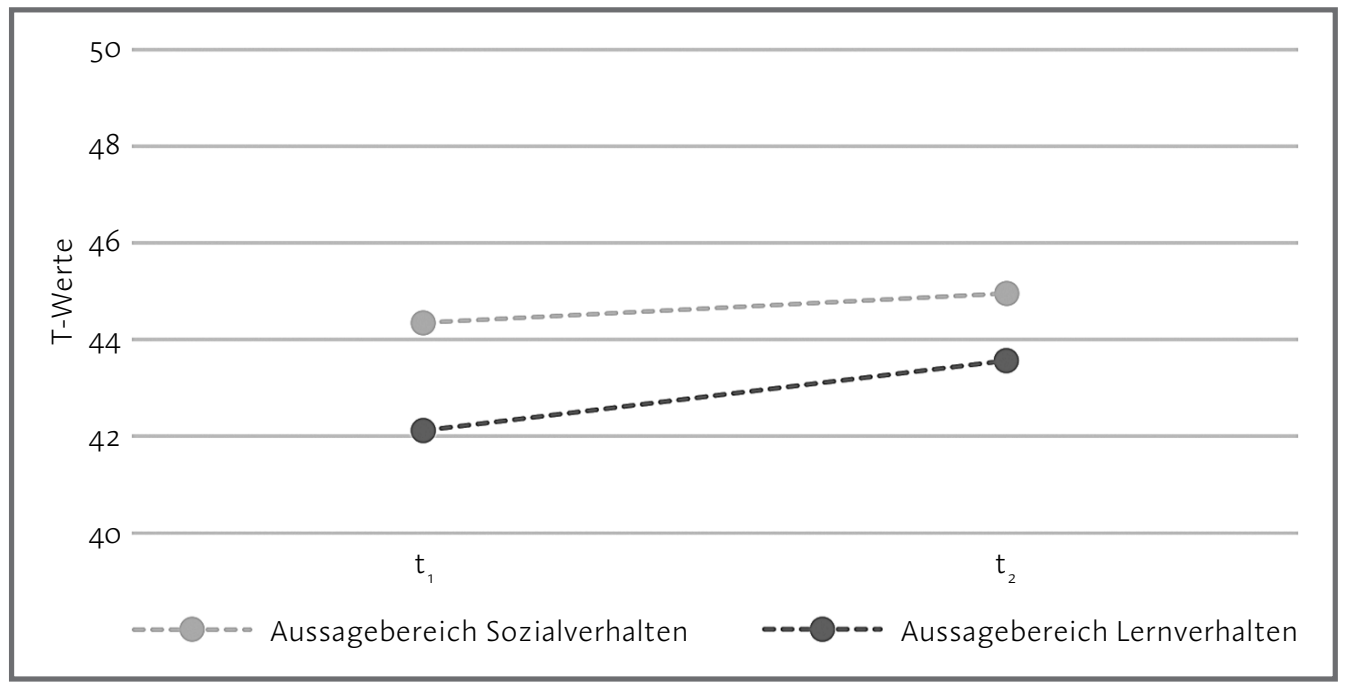

Abb. 4 Veränderung der Aussagebereiche Sozial- und Lernverhalten über den Untersuchungszeitraum (T-Werte im Mittel, $N=65$ ) 
$N=65)$. Sowohl zu Studienbeginn $(M=44.35$, $S D=6.80)$ als auch in der Folgebefragung $(M=44.95, S D=7.10)$ lag der mittlere T-Wert der gesamten Stichprobe auf etwa gleichem Niveau (s. Abb. 4).

Auch in den meisten Subgruppen zeigten sich keine bedeutsamen Veränderungen des Sozialverhaltens während des Untersuchungszeitraumes. Eine Ausnahme bildete die Gruppe der 10- bis 19-jährigen Schüler/innen. Die Untersuchungsergebnisse mittels $t$-Test zeigten für diese Gruppe eine signifikant positive Entwicklung der T-Werte $(t=-2.045, p=.048, n=37)$ zwischen $\mathrm{t}_{1}$ und $\mathrm{t}_{2}$ (vgl. Tab. 4). Zu Messzeitpunkt $\mathrm{t}_{2}(M=46.96, S D=6.87)$ fiel die Lehrer/inneneinschätzung im Aussagebereich Sozialverhalten signifikant besser aus als zu Messzeitpunkt $\mathrm{t}_{1}(M=45.36, S D=6.90)$. Die Effektstärke lag bei $r=.32$ und entsprach damit einem mittleren Effekt.

\subsubsection{Subskalen und Subgruppen im Aussagebereich Sozialverhalten}

Die sechs Subskalen (Kooperation, Selbstwahrnehmung, Selbstkontrolle, Einfühlungsvermögen und Hilfsbereitschaft, angemessene Selbstbehauptung, Sozialkontakt) bildeten den Ausgangspunkt weiterführender Analysen.

Die T-Werte in den Subskalen erwiesen sich überwiegend als zeitlich stabil sowohl in der Gesamtstichprobe (s. Abb. 5) als auch in den Subgruppen. Hinweise auf signifikante Veränderungen während des Untersuchungszeitraumes fanden sich lediglich bei den 10- bis 19-jährigen Schüler/innen in der Subskala Sozialkontakt (vgl. Tab. 4). Die Klassenlehrkräfte schätzten den Sozialkontakt der 10- bis 19-jährigen Schüler/innen zum Zeitpunkt $\mathrm{t}_{2}$ $(M d=45)$ signifikant höher ein als zum Zeitpunkt $\mathrm{t}_{1}(M d=44$; asymptotischer WilcoxonTest: $z=-2.099, p=.036, n=36$ ). Die Effektstärke nach Cohen (1988) lag bei $r=.34$ und entsprach damit einem mittleren Effekt.

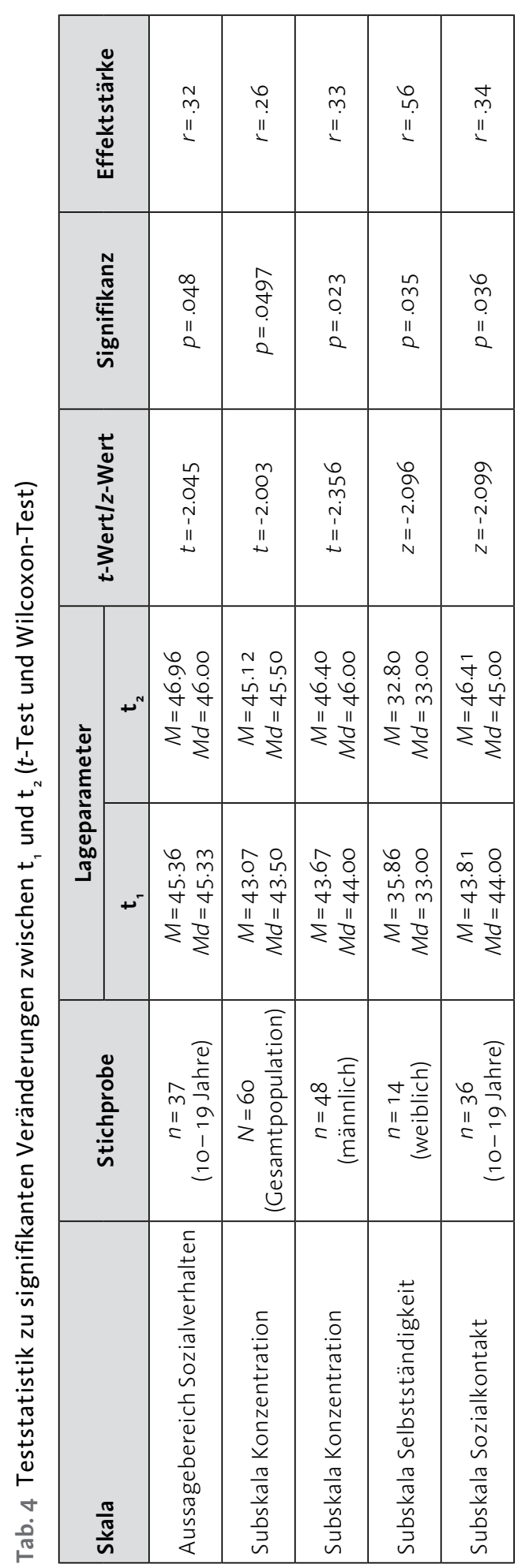




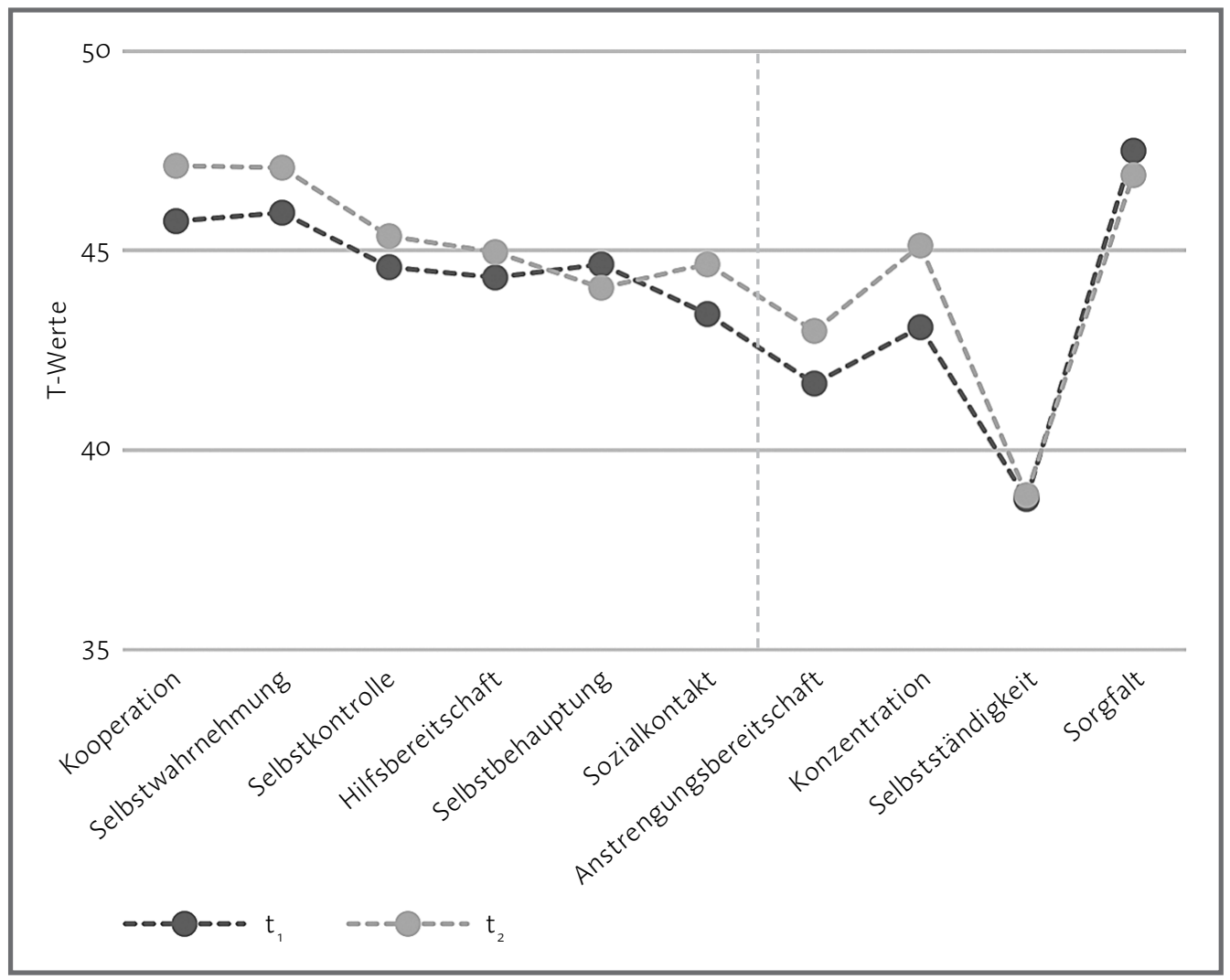

Abb. 5 Veränderung der Subskalen über den Untersuchungszeitraum (T-Werte im Mittel, $N=65$ )

Entwicklungstendenzen in positive Richtung - wenngleich ohne signifikantes Niveau - wurden daneben in den Subskalen Kooperation und Selbstkontrolle gefunden (vgl. Tab. 5). Die Lehrer/inneneinschätzung in der Subskala Kooperation (asymptotischer Wilcoxon-Test: $z=-1.748, p=.080, N=62)$ für die gesamte Stichprobe fiel in der Folgebefragung $(M d=47)$ positiver aus als in der Erstbefragung $(M d=46)$. Gleiches gilt für die 10- bis 19-Jährigen: Auch hier schätzten die Lehrer/innen die Kooperationsfähigkeit (asymptotischer Wilcoxon-Test: $z=-1.700, p=.089, n=37)$ zu Messzeitpunkt $\mathrm{t}_{2}$ $(M d=50)$ positiver ein als zu $\mathrm{t}_{1}(M d=44)$. Bei Jungen mit Schulassistenz fanden sich darüber hinaus Hinweise auf Fortschritte im Bereich Selbstkontrolle (asymptotischer Wilcoxon-
Test [2-seitig]: $z=-1.806, p=.071, n=47)$. Diese wurde von den Lehrer/innen in der Folgebefragung $(M d=46)$ positiver eingeschätzt als in der Erstbefragung $(M d=44)$. Mit Untersuchungsergebnissen von $p>.05$ waren die Unterschiede zwar nicht signifikant, jedoch deuten sie auf positive Entwicklungen in den Bereichen Kooperation und Selbstkontrolle hin.

Bei Betrachtung der Entwicklung des Sozialverhaltens vor dem Hintergrund des Lernortes zeigten sich weder bei Schüler/innen der allgemeinen Schule noch bei Schüler/innen an Förderschulen Hinweise auf signifikante Unterschiede zwischen den Messzeitpunkten in den Subskalen des Sozialverhaltens. 
FLORIAN SCHINDLER Lern- und Sozialverhalten bei Schülerinnen und Schülern mit Schulassistenz

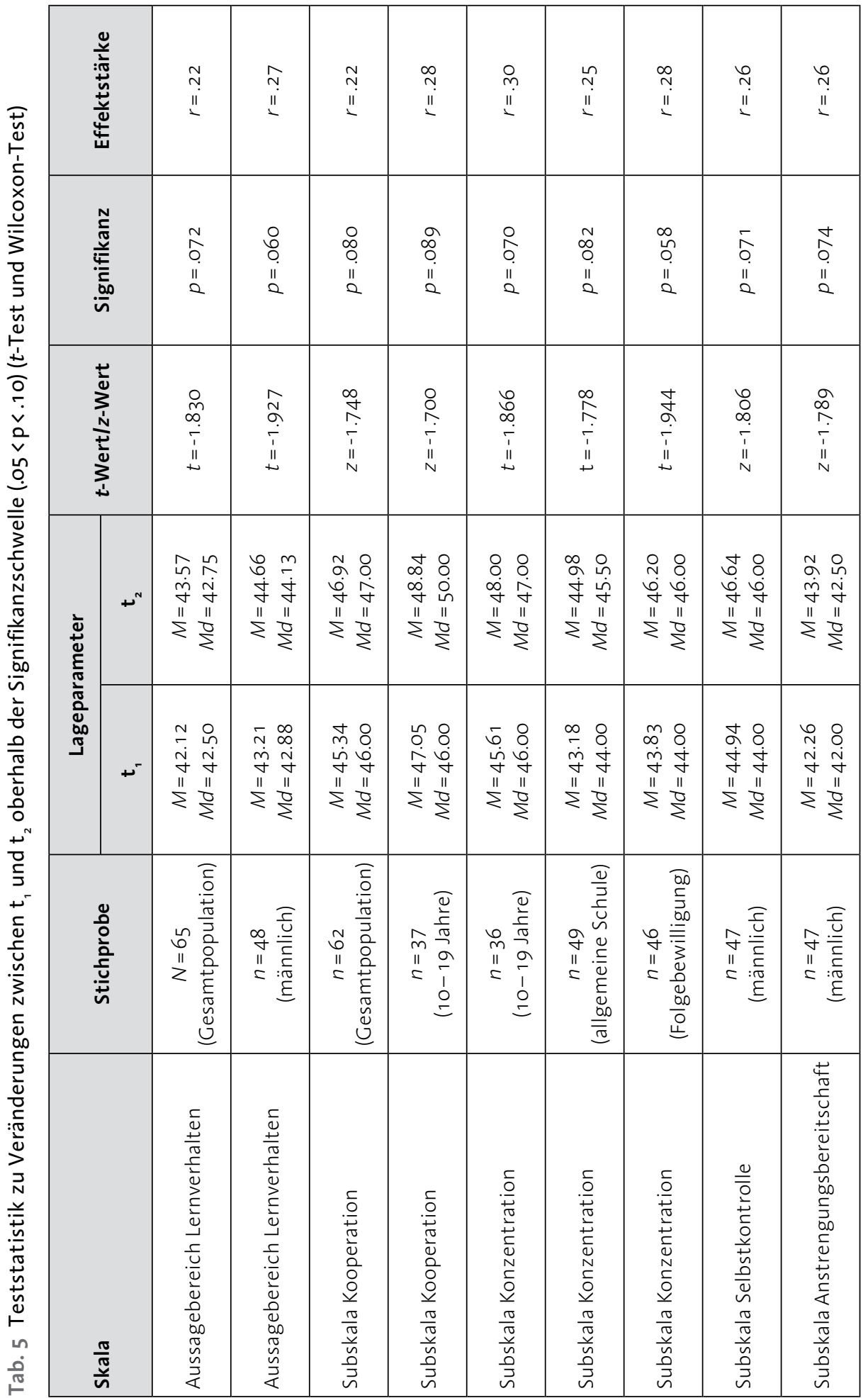




\subsubsection{Aussagebereich Lernverhalten}

Im Aussagebereich Lernverhalten kam es während des Untersuchungszeitraumes zu keiner signifikanten Veränderung $(t=-1.830, p=.072$, $N=65)$. Obgleich der statistische Beleg für eine signifikante Veränderung zwischen Erstbefragung $(M=42.12, S D=7.23)$ und Folgebefragung $(M=43.57, S D=7.27)$ nicht erbracht werden konnte, deutet das Untersuchungsergebnis mit $p=.07>.05$ auf eine tendenziell positive Gesamtentwicklung begleiteter Schüler/innen im Bereich Lernverhalten hin (vgl. Abb. 4).

\subsubsection{Subskalen und Subgruppen} im Aussagebereich Lernverhalten

Der Aussagebereich Lernverhalten gliedert sich in die vier Subskalen Anstrengungsbereitschaft und Ausdauer, Konzentration, Selbstständigkeit beim Lernen und Sorgfalt beim Lernen.

Für die Analyse von Veränderungen zwischen Erst- und Folgebefragung wurde zunächst die gesamte Stichprobe mit $N=65$ Fällen betrachtet. Die Untersuchungsergebnisse zeigen Hinweise auf eine bedeutsame Veränderung der Konzentrationsleistung während des Untersuchungszeitraumes. Die Klassenlehrkräfte schätzten die Konzentrationsleistung der Schüler/innen mit Schulassistenz zu Zeitpunkt $\mathrm{t}_{2}(M=45.12, S D=9.24)$ signifikant positiver ein als zu Zeitpunkt $\mathrm{t}_{1}(M=43.07, S D=8.74$; $t=-2.003, p=0.0497, N=60)$. Die Effektstärke entsprach mit $r=.25$ einem kleinen bis mittleren Effekt. Zur Absicherung der Analyse wurde zusätzlich der voraussetzungsärmere Wilcoxon-Test gerechnet. Die Testergebnisse stützen die Annahme einer signifikant positiven Veränderung der Konzentrationsleistung zwischen $\mathrm{t}_{1}(M d=43.5)$ und $\mathrm{t}_{2}(M d=45$; asymptotischer Wilcoxon-Test: $z=-2.061, p=.039$, $N=60)$ mit einer kleinen bis mittleren Effektstärke $(r=.26)$.
Weitere relevante Veränderungen zwischen Erstbefragung und Folgebefragung wurden bei geschlechtsspezifischer Betrachtung gefunden. Bei Mädchen mit Schulassistenz zeigte der Wilcoxon-Test eine signifikante Veränderung in der Subskala Selbstständigkeit (exakter Wilcoxon-Test: $z=-2.096, p=.035, n=14)$. Die Selbstständigkeit zu Zeitpunkt $t_{2}$ wurde von den Klassenlehrkräften signifikant negativer eingeschätzt als zu Zeitpunkt $t_{1}$. Der mittlere T-Wert zu Zeitpunkt $\mathrm{t}_{2}$ lag bei $M=32.80$ $(S D=3.570, M d=33)-\mathrm{zu} \mathrm{t}_{1}$ bei $M=35.86$ $(S D=7.533, M d=33)$. Der Befund wird durch die Rangverteilung gestützt: 7 negativen Rängen stehen 3 positive Ränge gegenüber. Die Effektstärke betrug $r=.56$ (starker Effekt). Bei Jungen mit Schulassistenz wurde mittels $t$-Test eine signifikante Verbesserung der Konzentrationsleistung nachgewiesen. Die Klassenlehrkräfte schätzten die Konzentration der männlichen Schüler zu Zeitpunkt $\mathrm{t}_{2}(M=46.40$, $S D=8.560)$ signifikant höher ein als zu Zeitpunkt $\mathrm{t}_{1}(M=43.67, S D=8.847 ; t=-2.356$, $p=.023, n=48)$. Die Effektstärke lag bei $r=.33$ (mittlerer Effekt). Sowohl für die Gesamtstichprobe wie auch für die Subgruppe der männlichen Schüler wurde eine Konzentrationssteigerung nachgewiesen. Mit einer Überschreitungswahrscheinlichkeit von $p=.023$ und einer Effektstärke von $r=.33$ erweist sich der Zugewinn an Konzentrationsleistung bei Jungen jedoch als vergleichsweise bedeutsamer.

Im Folgenden werden Ergebnisse berichtet, bei denen die Signifikanzschwelle knapp verfehlt wurde (vgl. Tab. 5). Sowohl bei Schüler/innen an allgemeinen Schulen als auch bei Schüler/innen mit Folgebewilligung und in der Gruppe der 10- bis 19-Jährigen wurden Hinweise auf positive Entwicklungstendenzen im Bereich Konzentration gefunden. Die Lehrer/inneneinschätzung in der Subskala Konzentration $(t=-1.778, p=.082, n=49)$ fiel bei Schüler/innen, die eine allgemeine Schule besuchten, in der Folgebefragung $(M=44.98$, $S D=9.48)$ positiver aus als in der Erstbefra- 
gung ( $M=43.18, S D=8.73)$. Gleiches gilt für die Gruppe der Schüler/innen mit Folgebewilligung $(t=-1.944, p=.058, n=46)$. Diese konnten während des Untersuchungszeitraumes von $\mathrm{t}_{1}(M=43.83, S D=7.95) \mathrm{zu} \mathrm{t}_{2}(M=46.20$, $S D=9.36$ ) ebenfalls Zugewinne im Bereich der Konzentrationsleistung verzeichnen. Auch bei den 10- bis 19-Jährigen fiel die Lehrer/inneneinschätzung in der Subskala Konzentration $(t=-1.866, p=0.70, n=36)$ in der Folgebefragung $(M=48.00, S D=8.89)$ positiver aus als in der Erstbefragung $(M=45.61, S D=7.80)$. Bei den männlichen Schülern fanden sich darüber hinaus Hinweise auf Fortschritte im Aussagebereich Lernverhalten und in der Subskala Anstrengungsbereitschaft. Das Lernverhalten ( $t=-1.927, p=0.60, n=48)$ wurde von den Klassenlehrkräften in der Folgebefragung $(M=44.66, S D=6.89)$ tendenziell positiver eingeschätzt als in der Erstbefragung $(M=43.21$, $S D=7.20$ ). Gleiches gilt für die Subskala Anstrengungsbereitschaft (asymptotischer Wilcoxon-Test (2-seitig): $z=-1.789, p=.074, n=47$ ). Auch hier kamen die Klassenlehrkräfte in der Folgebefragung $(M d=42.5)$ zu einer positiveren Einschätzung als in der Erstbefragung $(M d=42)$. Trotz Überschreitung der Signifikanzschwelle unterstützen die vorliegenden Ergebnisse den Gesamteindruck positiver Entwicklungstendenzen im Aussagebereich Arbeitsverhalten und einigen seiner Subskalen.

Abschließend wird die Entwicklung des Lernverhaltens vor dem Hintergrund des Lernortes betrachtet. Weder bei Schüler/innen, die eine Förderschule besuchten, noch bei jenen, die eine allgemeine Schule besuchten, fanden sich Hinweise auf signifkante Unterschiede zwischen den Messzeitpunkten.

\section{Diskussion}

Das Ziel des vorliegenden Beitrags war es, das Lern- und Sozialverhalten von Schüler/innen mit Schulassistenz über den Zeitraum eines
Schuljahres zu untersuchen. Erstmals im deutschsprachigen Raum wurden hierbei Entwicklungsverläufe im Längsschnitt mit einem standardisierten Verfahren erfasst. Hierzu wurden Lehrer/inneneinschätzungen der betreffenden Schüler/innen im Herbst und im darauffolgenden Frühjahr eines Schuljahres erhoben. Die Analysen lieferten deskriptive Erkenntnisse sowie detaillierte Einblicke in die Entwicklung des Lern- und Sozialverhaltens der betrachteten Schüler/innenpopulation $(N=65)$. Subgruppenanalysen ermöglichten darüber hinaus interessante Einblicke in Entwicklungen z. B. von Mädchen oder von älteren Schüler/innen mit Schulassistenz.

\subsection{Schülerlinnen mit Schulassistenz - deskriptive Erkenntnisse}

Die Leistungsempfänger von Schulassistenz an allgemeinen Schulen wiesen mit großer Mehrheit zusätzlich einen sonderpädagogischen Unterstützungsbedarf auf, obgleich dieser keine notwendige Bedingung für die Gewährung einer Eingliederungshilfe darstellt (vgl. Thiel, 2017). Hierin spiegelt sich der verbreitet kompensatorische Charakter von Schulassistenz wider (Henn et al., 2014): Hohe individuelle und ggf. sonderpädagogische Unterstützungsbedarfe werden durch Schulassistenz kompensiert, wenn die Passung zwischen Lernvoraussetzungen von Kindern und Jugendlichen und der konzeptionellen Ausgestaltung des Bildungsortes unzureichend ist (Dworschak, 2017). Henn et al. (2014, S. 401) halten fest: „Ein oftmals vorgebrachter Kritikpunkt zu Schulbegleitern [besteht darin], sie seien durch die Sozialsysteme finanzierte ,Ausfallbürgen für das Schulsystem". Dabei stellt sich die Frage, inwiefern der Grundgedanke von Inklusion an allgemeinen Schulen umgesetzt wird: ob das System Schule sich wirklich an die Bedürfnisse der Lernenden anpasst (vgl. Dworschak, 2017) - oder vielmehr versucht, Kinder und Jugendliche durch Schulassistenz an das Sys- 
tem anzupassen (ebd.). Dworschak (2017) warnt in diesem Zusammenhang, dass ,Schulbegleitung auch keinesfalls als die ideale Lösung für die Entwicklung eines inklusiven Bildungssystems angesehen werden“ (ebd., S. 48) darf. Die vorliegende Studie kann jedoch, ebenso wie die Studie von Henn et al. (2014), diese Befürchtungen nicht entkräften.

Zudem zeigte sich die überraschende Erkenntnis, dass der Anteil der Förderschüler/innen, die in Förderschulen unterrichtet wurden $(20 \%)$, deutlich unter der in NRW üblichen Förderschulquote von 60 Prozent lag. Dies deutet darauf hin, dass Schulassistenz in erster Linie an allgemeinen Schulen zum Einsatz kommt. Aktuelle Studien zum Einsatz von Schulassistenz an allgemeinen Schulen stützen diesen Eindruck (vgl. z. B. Dworschak, 2015). Der Anteil männlicher Schüler in der Stichprobe $(74 \%)$ war hingegen erwartungsgemäß hoch und reflektiert das allgemeine Übergewicht männlicher Schüler im Bereich sonderpädagogischer Förderung (vgl. Malecki, 2016).

Die vorliegenden Ergebnisse zu Altersstrukturen weisen darauf hin, dass die Erstversorgung mit Schulassistenz vorwiegend im Grundschulalter erfolgt. Dies spiegelt das schulische Bemühen um Ausschöpfung der verfügbaren Unterstützungsmöglichkeiten in den ersten Schuljahren wider. Gerade hier ist aufgrund der Bestimmungen zur Feststellungsdiagnostik bei Lern- und Entwicklungsstörungen (vgl. Schulministerium NRW, 2016) der Zugang zu sonderpädagogischen Ressourcen begrenzt - zudem herrscht derzeit generell eine Unterversorgung mit Sonderpädagogen (vgl. Klemm, 2012; Benus \& Weidemann, 2016). Schulassistenz scheint ergänzend und teilweise womöglich auch mit dem Ziel der Kompensation fehlender sonderpädagogischer Ressourcen gerade bei inklusiver Beschulung zum Einsatz zu kommen (vgl. Heinrich \& Lübeck, 2013).
Schulassistenz ist als zeitlich begrenzte Maßnahme konzipiert, welche mit dem Ziel erfolgt, die gewährte Hilfeleistung sukzessiv abzubauen (Henn et al., 2014) und begleitete Schüler/innen zunehmend in die Eigenständigkeit zu entlassen (Meyer, 2017). Der vorliegende Datensatz deutet auf einen solchen Trend abnehmender Bewilligungszahlen mit zunehmendem Alter hin (vgl. Abb. 1). Die ungleiche Verteilung der Fallzahlen über das gegliederte Schulsystem schließt an die von Klemm und Preuß-Lausitz (2011) dargestellte Ungleichverteilung bei der Umsetzung der schulischen Inklusion an.

Die vorliegende Studie bestätigt, dass Schulassistenz stärker von Jungen als von Mädchen in Anspruch genommen wird (vgl. Dworschak, 2015). Neu sind in diesem Zusammenhang Erkenntnisse zu geschlechtsspezifischen Unterschieden bei Betrachtung unterschiedlicher Altersgruppen. Bei Mädchen betrafen Unterstützungsleistungen aus dem Bereich Schulassistenz eher die frühe und mittlere Kindheit, während Jungen Schulassistenz eher in der späten Kindheit und Adoleszenz erhielten. Dieser Unterschied ist möglicherweise durch die Prävalenz und den Ausdruck psychischer Auffälligkeiten zu erklären. Jungen sind deutlich häufiger von psychischen Problemen betroffen als Mädchen (Hölling, Schlack, Petermann, Ravens-Sieberer \& Mauz, 2014) und äußern diese zudem vorwiegend externalisierend (Hölling, Erhart, Ravens-Sieberer \& Schlack, 2007). Im Schulalltag gehen hiermit stärkere Belastungen einher. Jungen werden daher vermutlich nicht nur häufiger, sondern auch länger mit Schulassistenz versorgt als Mädchen.

Die Schüler/innen mit Schulassistenz erreichten über alle Subskalen hinweg im Mittel Rohwerte, die knapp $30 \%$ unter denen der Eichstichprobe (LSL, vgl. Petermann \& Petermann, 2013) lagen (s. Abb. 3). Während die Abweichung im Aussagebereich Sozial- 
verhalten mittelgroß war, wichen die Werte im Lernverhalten teilweise erheblich nach unten ab. Am deutlichsten betraf dies die Subskala Selbstständigkeit: Die Entwicklung war in diesem Bereich nicht nur durch einen negativen Entwicklungsverlauf gekennzeichnet, die erreichten Rohwertpunkte deuten auch auf eine massiv eingeschränkte Selbstständigkeit der Schüler/innen mit Schulassistenz hin (vgl. Abschnitt 5.3). Dies wirft die Frage auf, wie es gelingen kann, selbstständigkeitsförderliche Rahmenbedingungen für Schüler/innen mit Schulassistenz zu schaffen.

Ein entgegengesetzter Trend wurde in der Subskala Konzentration beobachtet. Hier verringerte sich die Differenz zur Eichstichprobe über den Untersuchungszeitraum deutlich. Damit stellt die Konzentrationsleistung jenen Bereich des Lernverhaltens dar, in dem sich die Schüler/innen mit Schulassistenz gegenüber der Eichstichprobe am meisten steigerten - die signifikanten Effekte bei der Entwicklung der Konzentrationsleistung untermauern dies (s. Abschnitt 5.3).

Die betrachtete Schüler/innenpopulation ist insgesamt gekennzeichnet durch ein schwaches Lern- und Sozialverhalten. Es ist anzunehmen, dass die Abweichungen im Lern- und Sozialverhalten und die hiermit einhergehenden Einschränkungen der schulischen Leistungsfähigkeit (vgl. Petermann \& Petermann, 2013) eine wichtige Rolle bei der Beantragung von Schulassistenz spielen.

\subsection{Entwicklungen im Sozialverhalten}

Das Sozialverhalten der Schüler/innen mit Schulassistenz erwies sich insgesamt als stabil mit tendenziell positivem Entwicklungsverlauf, v.a. in der späten Kindheit und Jugend (10 - 19 Jahre). Da die LSL zur Verlaufsbeurteilung empfohlen wird (vgl. Petermann \& Peter- mann, 2013) und spontane Reifungsprozesse anhand von Altersnormen weitgehend kontrolliert werden können, scheint dieses Ergebnis darauf hinzudeuten, dass Schulassistenz vor dem Hintergrund der hohen Bedeutung des Sozialverhaltens für Schulleistung und Schulerfolg (Moilanen et al., 2010; Romano et al., 2010) - während der Sekundarstufe einen Beitrag zu einem erfolgreichen Schulbesuch leisten kann.

Nach Einschätzung der Klassenlehrkräfte lagen die Leistungen der Schüler/innen mit Schulassistenz in den Subskalen des Sozialverhaltens zu beiden Erhebungszeitpunkten durchgängig im unteren Durchschnittsbereich (s. Abb. 5), entwickelten sich jedoch tendenziell positiv - mit signifikanten Effekten in der Subskala Sozialkontakt bei den 10- bis 19-jährigen. Die auffällige Steigerung im Bereich Sozialkontakt ist überraschend, da Schulassistenz als Einzelfallhilfe oftmals mit Schwierigkeiten in der sozialen Partizipation assoziiert wird (vgl. Böing, 2014; Lücke \& Ehrenberg, 2017). Die vorliegende Studie kann somit aufzeigen, dass Schulassistenz bei älteren Kindern und Jugendlichen nicht $\mathrm{zu}$ Vereinzelung führen muss, sondern mit einer Normalisierung sozialer Kontakte einhergehen kann. Diese positiven Entwicklungsverläufe sind möglicherweise Ausdruck zunehmender Eigenständigkeit und Verselbstständigung, wie sie von Schulassistenz als wichtiges Ziel langfristig angestrebt werden (vgl. Henn et al., 2014; Sommer, Czempiel, Kracke \& Sasse, 2017).

Nicht zuletzt wurde auch die Kooperation von Schüler/innen mit Schulassistenz von den Lehrkräften über den Untersuchungszeitraum hinweg als verbessert wahrgenommen. Obwohl die Signifikanzschwelle knapp verfehlt wurde, ist die Entwicklung in diesem Bereich als positiv zu bewerten. Hier wäre eine Studie mit größeren Fallzahlen wünschenswert, um die angedeuteten Entwicklungsverläufe genauer zu untersuchen. 
Anhand der vorliegenden Daten wurden relevante Entwicklungsverläufe im Sozialverhalten lediglich für die Subgruppe der 10 bis 19 Jahre alten Schüler/innen beobachtet. Auch bei lernortspezifischer Betrachtung konnten keine Veränderungen identifiziert werden. Entwicklungen im Sozial- und Lernverhalten (s. Abschnitt 5.3) sind offenbar weniger abhängig vom Lernort (Förderschule vs. allgemeine Schule) als von anderen Faktoren wie bspw. Qualifikationen von Leistungserbringern (vgl. Geist, 2017) oder der Qualität des Hilfeplans (vgl. Bundesarbeitsgemeinschaft Landesjugendämter, 2015).

\subsection{Entwicklungen im Lernverhalten}

Erstmals wurde in dieser Untersuchung die Entwicklung des Lernverhaltens aus Sicht der Klassenlehrkräfte erfasst. Hierbei ergaben sich Hinweise auf positive Entwicklungsprozesse für das Lernverhalten insgesamt und die Konzentrationsleistung im Speziellen.

Die Konzentrationsleistung war in der vorliegenden Studie der einzige Teilbereich des Lernund Sozialverhaltens, für den sich über die gesamte Stichprobe hinweg eine signifikante Verbesserung zeigte. Bei männlichen Schülern war diese Verbesserung besonders ausgeprägt. Dies bestätigt die Erkenntnisse aus dem Vergleich der betrachteten Schüler/innenpopulation mit der Eichstichprobe (s. Abschnitt 5.1). Schulassistenz scheint sich positiv auf die Konzentrationsleistung von Schüler/innen auszuwirken, worauf bereits die Befunde von Zauner und Zwosta (2014) hinweisen. Dies würde dem Anliegen von Schulassistenz in hohem Maße entsprechen, tragen doch positive Entwicklungen in den Bereichen Aufmerksamkeit und Konzentration dazu bei, Schulerfolg und Teilhabe am Lernen sicherzustellen (vgl. Ladd et al., 1999). Zudem stellen Schwierigkeiten in der Aufmerksamkeitssteuerung und Konzentration einen häufigen Antragsgrund für Eltern dar (vgl. Henn et al., 2014; Zauner \& Zwosta, 2014).
Im Widerspruch zu den Zielen von Schulassistenz stehen hingegen die Ergebnisse bezüglich der Selbstständigkeit begleiteter Schüler/innen. Hier zeigte sich insgesamt eine negative Entwicklung; für Mädchen war die Entwicklung über die sieben Untersuchungsmonate hinweg signifikant negativ. Schulassistenz scheint sich also nicht positiv auf die Selbstständigkeit von Schüler/innen auszuwirken. Auffällig ist, dass die Selbstständigkeitswerte der Mädchen mit Schulassistenz bereits zu Untersuchungsbeginn am unteren Ende der Skala lagen. Der negative Entwicklungsverlauf der Selbstständigkeitswerte ist möglicherweise durch kompensatorische Hilfen und Überbehütung im Zuge der Schulassistenz zu erklären. Giangreco, Edelman, Luiselli \& MacFarland (1997) sprechen in diesem Zusammenhang von hovering: Schulassistenz birgt demnach die Gefahr, Schüler/innen in eine Situation unnötiger Abhängigkeit von Erwachsenen zu bringen und die Entwicklung der Selbstständigkeit zu erschweren statt zu fördern. Künftige Forschung sollte diesen Sachverhalt genauer untersuchen und Ansatzpunkte liefern für eine selbstständigkeitsförderliche Unterstützung gerade von Mädchen mit Schulassistenz.

Während in einzelnen Subgruppen Veränderungen des Lernverhaltens beobachtet wurden, war dies bei lernortspezifischer Betrachtung nicht der Fall. Die Lernorte Förderschule und allgemeine Schule scheinen für die Entwicklung des Lern- und Sozialverhaltens (s. Abschnitt 5.2) nicht oder nur in sehr geringem Maße bedeutsam zu sein.

\subsection{Limitationen}

Bei der Interpretation der Ergebnisse müssen einige Einschränkungen beachtet werden. In der vorliegenden Studie wurde die Entwicklung der Schüler/innen mit Schulassistenz lediglich auf Ebene des Lern- und Sozialverhaltens erfasst. Es liegen somit allein Daten für 
zwei von vielen Faktoren für Schulerfolg vor. Entsprechend kann die vorliegende Studie keine Aussagen zur Wirkung von Schulassistenz auf Schulerfolg machen. Für ein vollständigeres Bild wäre eine umfassende und ganzheitliche Längsschnittuntersuchung nötig, die den Entwicklungsstand der Schüler/innen zu verschiedenen Zeitpunkten vor und nach der Erstbewilligung erfasst.

Auch die Erhebungsmethode - die Befragung der beteiligten Lehrkräfte - unterliegt naturgemäß Beschränkungen. So wurde das Lern- und Sozialverhalten in der vorliegenden Studie nicht selbst betrachtet, sondern dessen Wahrnehmung durch die Klassenlehrkräfte. Diese subjektiven Einschätzungen unterliegen stets einer gewissen Anfälligkeit für Verzerrungen. Für ein ganzheitlicheres Bild wäre eine mehrperspektivische Betrachtung, die z. B. die Einschätzungen von Eltern, Schulassistent/innen und Schüler/innen selber einschließt, angezeigt. Um das Lern- und Sozialverhalten möglichst differenziert zu erfassen, sollte künftige Forschung bspw. auch Beobachtungen im Klassenkontext bei Anwesenheit und Abwesenheit der Schulassistenz mit einbeziehen.

Bezogen auf das vorliegende Studiendesign ist der überschaubare Stichprobenumfang von $N=65$ als Einschränkung zu nennen. Eine größere Stichprobe wäre für die Identifikation relevanter Entwicklungsverläufe im Lern- und Sozialverhalten vorteilhaft gewesen. Obwohl es sich bei der Stichprobe um eine Zufallsstichprobe handelte, lässt der vorliegende Datensatz nur bedingt Verallgemeinerungen zu. Für die Zukunft wären Studien mit größeren Datensätzen sinnvoll. Zudem wäre es vorteilhaft, mehr Schüler/innen mit beginnender Schulassistenz zu untersuchen. In der vorliegenden Studie wurde beides - ein größerer Datensatz sowie v. a. eine höhere Anzahl von Schüler/innen mit beginnender Schulassistenz - angestrebt, konnte jedoch aufgrund der besonderen Bedingungen im Zuge der Beantragung und Bewilligung von Schulassistenz nicht realisiert werden. Die relativ kleine Subgruppe von 14 Schüler/innen mit Erstbewilligung von Schulassistenz war nicht groß genug, um valide bedeutsame Entwicklungen erfassen zu können. Auch ein Kohortenvergleich zwischen Schüler/innen mit Erst- und Folgebewilligung konnte aus den genannten Gründen nicht realisiert werden.

Eine weitere Limitation betrifft die Analysen im vorliegenden Beitrag, v. a. die Subgruppenanalysen. Subgruppenanalysen bergen das Problem der Multiplizität und bei steigender Anzahl die Gefahr falsch-positiver Ergebnisse (Schulz \& Grimes, 2005). In der vorliegenden Studie mit ihrem explorativen Charakter (vgl. Goeman \& Solari, 2011) wurde jedoch - auch vor dem Hintergrund der Herausforderung der Teilnehmerakquise - nicht darauf verzichtet, Subgruppenanalysen durchzuführen, denn die statistischen Analysen eröffnen einen ersten Eindruck zur Entwicklung wesentlicher schulbezogener Merkmale und Kompetenzen von Schüler/innen mit Schulassistenz. Die Ergebnisse sind jedoch keineswegs als Endprodukte zu verstehen, sondern sollten in unabhängigen Validierungsstudien aufgegriffen und dort untersucht werden. Bedingt durch die potentielle Schwierigkeit der Multiplizität und die Größe des Datensatzes wurden in der vorliegenden Studie teilweise weitergehende Subgruppenanalysen unterlassen, wie z. B. eine Analyse der Subgruppen nach Förderschwerpunkten. Hiermit wurde data dredging (Schulz \& Grimes, 2005) vermieden. Auch wenn die durchgeführten Subgruppenanalysen mit ihrem explorativen Charakter erste wichtige Hinweise auf Entwicklungstendenzen begleiteter Schüler/innen liefern, gilt es, diese zurückhaltend $\mathrm{zu}$ interpretieren, nur mit Vorsicht Schlussfolgerungen zu ziehen (Schulz \& Grimes, 2005) und stattdessen als zuverlässigsten Effekt den Gesamteffekt - in diesem Fall die Entwicklungen des Lern- und Sozialverhaltens sowie seiner Teilbereiche - zu betrachten (Yusuf, Wittes, Probstfield \& Tyroler, 1991). 


\section{Anmerkung}

Die unveröffentlichte Diplomarbeit ist auf Anfrage nicht erhältlich. Informationen zur Studie stammen aus Mitteilungen von W. Dworschak (15.4.2018).

\section{Literatur}

Ansary, N.S. \& Luthar, S.S. (2009). Distress and academic achievement among adolescents of affluence: A study of externalizing and internalizing problem behaviors and school performance. Development and Psychopathology, 21 (1), 319-341. https://doi.org/10.1017/s0954 579409000182

Asendorpf, J.B. \& Neyer, F.J. (2012). Psychologie derPersönlichkeit. Berlin: Springer.

Bacher, J., Pfaffenberger, M. \& Pöschko, H. (2007). Arbeitssituation und Weiterbildungsbedarfvon Schulassistent/innen. Endbericht. Korrigierte Fassung. Abgerufen am 2.1.2019 von http://www. forschungsnetzwerk.at/downloadpub/schul assistenz_jugendliche_mit_foerderbedarf_oo e_2007.pdf

Beck, C., Dworschak, W. \& Eibner, S. (2010). Schulbegleitung am Förderzentrum mit dem Förderschwerpunkt geistige Entwicklung. Zeitschrift für Heilpädagogik, 61 (7), 244-254.

Benus, U. \& Weidemann, G. (2016). Lehrkräftebedarf, Abordnungen und Versetzungen im Gemeinsamen Lernen: Der Unmut wächst. NDS Die Zeitschrift der Bildungsgewerkschaft (8), $10-11$.

Böing, U. (2014). Die soziale Situation blinder und sehbeeinträchtigter Schülerinnen und Schüler in inklusiven Settings. Anfragen an die Rolle der Integrationshilfe. Gemeinsam leben, 22 (4), 219-225.

Böing, U. \& Köpfer, A. (2017). Schulassistenz aus der Sicht der Schülerinnen und Schüler mit Assistenzerfahrung. In M. Laubner, B. Lindmeier \& A. Lübeck (Hrsg.), Schulbegleitung in der inklusiven Schule. Grundlagen und Praxishilfen, 127-136. Weinheim: Beltz. https://doi.org/10. 2378/vhn2018.art29d

Bortz, J. \& Lienert, G. A. (2008). Kurzgefasste Statistik für die klinische Forschung. Heidelberg: Springer. https://doi.org/10.1007/978-3-662-22 o78-8
Budde, J. (2012). Die Rede von der Heterogenität in der Schulpädagogik. Diskursanalytische Perspektiven. Forum Qualitative Sozialforschung, 13(2). Abgerufen am 22.4.2018 von http:/l www.qualitative-research.net/index.php/fqs/ article/view/1761/3358

Bundesarbeitsgemeinschaft Landesjugendämter (Hrsg.) (2015). Qualitätsmaßstäbe und Gelingensfaktoren für die Hilfeplanung gemäß $\S 36$ SGB VIII. Abgerufen am 2.1.2019 von http:II www.bagljae.de/downloads/123_hifelplanunggem.-36-sgb-viii_2015.pdf

Carroll, J.B. (1963). A model of school learning. Teachers College Record, 64 (8), 723-733.

Cohen, J. (1988). Statistical Power Analysis for the Behavioral Sciences. $2^{\text {nd }}$ ed. Hillsdale: Lawrence Erlbaum.

Dworschak, W. (2010). Schulbegleiter, Integrationshelfer, Schulassistent? Begriffliche Klärung einer Maßnahme zur Integration in die allgemeine Schule bzw. die Förderschule. Teilhabe, 49(3), $131-135$

Dworschak, W. (2012a). Schulbegleitung im Förderschwerpunkt geistige Entwicklung an der allgemeinen Schule. Ergebnisse einer bayerischen Studie im Schuljahr 2010/11. Gemeinsam Leben, 20(2), 80-94.

Dworschak, W. (2012 b). Schulbegleitung/Integrationshilfe. Ergebnisse einer Studie des Lebenshilfe-Landesverbandes Bayern. Abgerufen am 22.4. 2018 von https://epub.ub.uni-muenchen. de/13105/1/Dworschak_13105.pdf

Dworschak W. (2014a). Schulbegleitung - Die richtige Unterstützungsmaßnahme für Schüler mit (geistiger) Behinderung zur Realisierung ihres Bildungsrechts an der allgemeinen Schule? In B. Kopp et al. (Hrsg.), Individuelle Förderung und Lernen in der Gemeinschaft. Jahrbuch Grundschulforschung, 214-217. Wiesbaden: Springer. https://doi.org/10.1007/978-3-658-04479-4_37

Dworschak, W. (2014 b). Zur Bedeutung individueller Merkmale im Hinblick auf den Erhalt einer Schulbegleitung. Eine empirische Analyse im Förderschwerpunkt geistige Entwicklung an bayerischen Förderschulen. Empirische Sonderpädagogik, 6(2), 150-171.

Dworschak, W. (2015). Zur Bedeutung von Kontextfaktoren im Hinblick auf den Erhalt einer Schulbegleitung - Eine empirische Analyse im Förderschwerpunkt geistige Entwicklung an bayerischen Förderschulen. Empirische Sonderpädagogik, 7(1), 56-72. 
Dworschak, W. (2017). Zur Gewährung von Schulbegleitung - Wer erhält in welchem Umfang eine Schulbegleitung? In M. Laubner, B. Lindmeier \& A. Lübeck (Hrsg.), Schulbegleitung in der inklusiven Schule. Grundlagen und Praxishilfen, 37-49. Weinheim: Beltz.

Farrell, P., Alborz, A., Howes, A. \& Pearson, D. (2010). The impact of teaching assistants on improving pupils' academic achievement in mainstream schools: A review of the literature. Educational Review, 62 (4), 435-448. https://doi.org/10.108 oloo131911.2010.486476

Geist, E.M. (2017). Qualifikation und Qualifizierung von Schulbegleiter/innen. In M. Laubner, B. Lindmeier \& A. Lübeck (Hrsg.), Schulbegleitung in der inklusiven Schule. Grundlagen und Praxishilfen, 50-65. Weinheim: Beltz.

Giangreco, M.F., Edelman, S.W., Luiselli, T.E. \& MacFarland, S.Z.C. (1997). Helping or hovering? Effects of instructional assistant proximity on students with disabilities. Exceptional Children, 64(1),7-18. https://doi.org/10.1177/001440299 706400101

Goeman, J.J. \& Solari, A. (2011). Multiple testing for exploratory research. Statistical Science, 26(4), 584-597. https://doi.org/10.1214/11-sts 356

Hattie, J. (2008). Visible Learning: A Synthesis of Over 800 Meta-Analyses Relating to Achievement. London: Routledge. https://doi.org/10.43 24/9780203887332

Hattie, J. (2015). The applicability of visible learning to higher education. Scholarship of Teaching and Learning in Psychology, 1 (1), 79-91. https://doi.org/10.1037/stlooooo21

Heinrich, M. \& Lübeck, A. (2013). Hilflose häkelnde Helfer? Zur pädagogischen Rationalität von Integrationshelfer/inne/n im inklusiven Unterricht. Bildungsforschung, 10(1), 91-110.

Hellrung, C. (2016). Inklusion von Kindern mit Behinderungen als sozialrechtlicher Anspruch. Wiesbaden: Springer. https://doi.org/10.1007/ 978-3-658-16357-0

Henn, K., Thurn, L., Besier, T., Künster, A. K., Fegert, J.M. \& Ziegenhain, U. (2014). Schulbegleiter als Unterstützung von Inklusion im Schulwesen. Erhebung zur gegenwärtigen Situation von Schulbegleitern in Baden-Württemberg. Zeitschrift für Kinder-und Jugendpsychiatrie und Psychotherapie, 42(6), 397-403. https://doi. org/10.1024/1422-4917/a000318
Hinshaw, S.P. (1992). Externalizing behavior problems and academic underachievement in childhood and adolescence: causal relationships and underlying mechanisms. Psychological Bulletin, 111(1), 127-155. https://doi.org/ 10.1037/loo33-2909.111.1.127

Hölling, H., Erhart, M., Ravens-Sieberer, U. \& Schlack, R. (2007). Verhaltensauffälligkeiten bei Kindern und Jugendlichen. Erste Ergebnisse aus dem Kinder-und Jugendgesundheitssurvey (KiGGS). Bundesgesundheitsblatt - Gesundheitsforschung Gesundheitsschutz, 50(5-6), 784-793. https:/l doi.org/10.1007/s00103-007-0241-7

Hölling, H., Schlack, R., Petermann, F., Ravens-Sieberer, U. \& Mauz, E. (2014). Psychische Auffälligkeiten und psychosoziale Beeinträchtigungen bei Kindern und Jugendlichen im Alter von 3 bis 17 Jahren in Deutschland - Prävalenz und zeitliche Trends zu 2 Erhebungszeitpunkten (2003-2006 und 2009-2012). Ergebnisse der KiGGS-Studie - Erste Folgebefragung (KiGGS Welle 1). Bundesgesundheitsblatt - Gesundheitsforschung - Gesundheitsschutz, 57(7), 807-819. https://doi.org/10.1007/s00103-0141979-3

Kißgen, R., Franke, S., Ladinig, B., Mays, D. \& Carlitscheck, J. (2013). Schulbegleitung an Förderschulen in Nordrhein-Westfalen: Ausgangslage, Studienkonzeption und erste Ergebnisse. Empirische Sonderpädagogik, 4(3), 263-276.

Klemm, K. (2012). Zusätzliche Ausgaben für ein inklusives Schulsystem in Deutschland. Gütersloh: Bertelsmann Stiftung. Abgerufen am 22.4. 2018 von https://www.bertelsmann-stiftung. de/fileadmin/files/BSt/Publikationen/GrauePu blikationen/Zusaetzl_Ausgaben_inkl_Schulsys tem_in_D_Mrz_12.pdf

Klemm, K. \& Preuss-Lausitz, U. (2011). Auf dem Weg zur schulischen Inklusion in NordrheinWestfalen. Empfehlungen zur Umsetzung der UN-Behindertenrechtskonvention im Bereich der allgemeinen Schulen. Abgerufen am 25. 7. 2018 von https://www.schulministerium.nrw.de/docs/ Schulsystem/Inklusion/Lehrkraefte/Kontext/ Gutachten/NRW_Inklusionskonzept_2011_-neue_Version_08_07_11.pdf

Ladd, G.W., Birch, S. H. \& Buhs, E. S. (1999). Children's social and scholastic lives in kindergarten: Related spheres of influence? Child Development, 70(6), 1373-1400. https://doi.org/10.1111/14 67-8624.00101 
Lindmeier, B. \& Ehrenberg, K. (2017). „In manchen Momenten wünsch ich mir auch, dass sie gar nicht da sind" - Schulassistenz aus der Perspektive von Mitschülerinnen und Mitschülern. In M. Laubner, B. Lindmeier \& A. Lübeck (Hrsg.), Schulbegleitung in der inklusiven Schule. Grundlagen und Praxishilfen, 137-149. Weinheim: Beltz.

Lübeck, A. \& Demmer, C. (2017). Unüberblickbares überblicken - Ausgewählte Forschungsergebnisse zu Schulbegleitung. In M. Laubner, B. Lindmeier \& A. Lübeck (Hrsg.), Schulbegleitung in der inklusiven Schule, 11-27. Weinheim: Beltz.

Lücke, M. \& Ehrenberg, K. (2017). ,Der hat immer 'ne zweite Mutter bei sich' - Peerkontakte bei Schulassistenz aus der Perspektive von Grundschülerinnen und Grundschülern. Sonderpädagogische Förderung heute, 62 (1), 34-45.

Malecki, A. (2016). Schulen auf einen Blick. Wiesbaden: Statistisches Bundesamt. Abgerufen am 16.7. 2018 von https://www.destatis.de/DE/ Publikationen/Thematisch/BildungForschung Kultur/Schulen/BroschuereSchulenBlicko1 1 0018169004.pdf?__blob=publicationFile

Malmgren, K. W. \& Causton-Theoharis, J. N. (2006). Boy in the bubble: Effects of paraprofessional proximity and other pedagogical decisions on the interactions of a student with behavior disorders. Journal of Research in Childhood Education, 20(4), 301-312. https://doi.org/10.1080/ o2568540609594569

Markowetz, R. \& Jerosenko, A. (2016). Integrationshelfer in der inklusiven Schule. Abschlussbericht der wissenschaftlichen Begleitung - Projektlaufzeit:September 2013-Juli 2016. Abgerufen am 22.4. 2018 von http://bildungspakt-bayern. de/wp-content/uploads/2016/og/Abschlussbe richt_wissenschaftliche_Begleitung.pdf

McClelland, M.M., Morrison, F.J. \& Holmes, D.L. (2000). Children at risk for early academic problems: The role of learning-related social skills. Early Childhood Research Quarterly, 15 (3), 307329. https://doi.org/10.1016/so885-2006(oo)oo 069-7

Meyer, K. (2017). Multiprofessionalität in der inklusiven Schule: Eine empirische Studie zur Kooperation von Lehrkräften und Schulbegleiter/innen (Göttinger Schulbegleitungsstudie GötS). Abgerufen am 22.4. 2018 von https://www.univerlag. uni-goettingen.de/handle/3/issn-2198-238437

Moilanen, K. L., Shaw, D. S. \& Maxwell, K. L. (2010). Developmental cascades: Externalizing, inter- nalizing, and academic competence from middle childhood to early adolescence. Development and Psychopathology, 22 (3), 635-653. https://doi.org/10.1017/s0954579410000337

Newcomb, A. F., Bukowski, W.M. \& Pattee, L. (1993). Children's peer relations: A meta-analytic review of popular, rejected, neglected, controversial, and average sociometric status. Psychological Bulletin, 113(1), 99-128. https://doi. org/10.1037/loo33-2909.113.1.99

Oerter, R. \& Höfling, S. (2001). Zur Aktualität von Partizipation. In R. Oerter \& S. Höfling (Hrsg.), Mitwirkung und Teilhabe von Kindern und Jugendlichen, 9-13. München: Akademie für Politik und Zeitgeschehen, Hanns-Seidel-Stiftung.

Petermann, U. \& Petermann, F. (2013). Lehrereinschätzliste für Sozial- und Lernverhalten: LSL. Göttingen: Hogrefe. https://doi.org/10.1024/16 61-4747.55.3.209

Roberts, B.W. \& DelVecchio, W. F. (2000). The rankorder consistency of personality traits from childhood to old age: A quantitative review of longitudinal studies. Psychological Bulletin, 126 (1), 3-25. https://doi.org/10.1037//o033-2909. 126.1.3

Romano, E., Babchishin, L., Pagani, L. S. \& Kohen, D. (2010). School readiness and later achievement: Replication and extension using a nationwide Canadian survey. Developmental Psychology, 46(5), 995-1007. https://doi.org/10.1037/aoo1 8880

Schmidt, L.D.H. (2017). Schulische Assistenz - Der Forschungsstand in Deutschland mit Blick auf die internationale Ebene. Zeitschrift für Inklusion (4). Abgerufen am 22.4. 2018 von https:/I www.inklusion-online.net/index.php/inklu sion-online/article/view/372/312

Schulministerium NRW (Hrsg.): AO-SF (Stand: 1. 7. 2016). Abgerufen am 24.7.2018 von https:/l www.schulministerium.nrw.de/docs/Schulsys tem/Schulformen/Grundschule/Von-A-bis-ZI Ausbildungsordnung-sonderpaedagogischeFoerderung-_AO-SF_lindex.html

Schulz, K.F. \& Grimes, D.A. (2005). Multiplicity in randomised trials II: subgroup and interim analyses. The Lancet, 365 (9471), 1657-1661. https:/l doi.org/10.1016/s0140-6736(05)66516-6

Sharma, U. \& Salend, S.J. (2016). Teaching assistants in inclusive classrooms: A systematic analysis of the international research. Australian Journal of Teacher Education, 41 (8), 128-134. https://doi.org/10.14221/ajte.2016v41n8.7 
Sommer, S., Czempiel, S., Kracke, B. \& Sasse, A. (2017). Zwischen Einzelfallhelfer/in und Zweitlehrer/in: Wie handeln Integrationshelfer/innen im Unterricht? Diskurs Kindheits- und Jugendforschung, 12 (1), 35-47. https://doi.org/10.32 24/diskurs.v12i1.04

Symes, W. \& Humphrey, N. (2012). Including pupils with autisticspectrum disorders in the classroom: the role of teaching assistants. European Journal of Special Needs Education, 27(4), 517-532. https://doi.org/10.1080/08856257.20 12.726019

Thiel, S. (2017). Die Beantragung und Bewilligung von Schulassistenz. In M. Laubner, B. Lindmeier \& A. Lübeck (Hrsg.), Schulbegleitung in der inklusiven Schule. Grundlagen und Praxishilfen, 28-36. Weinheim: Beltz.

United Nations (2006). A Convention on the Rights of Persons with Disabilities. Abgerufen am 24.7. 2018 von http://www.un.org/disabilities/docu ments/convention/convention_accessible_pdf. pdf

Walberg, H.J. (1984). Improving the productivity of America's schools. Educational Leadership, 41 (8), 19-27.

Wang, M. C., Haertel, G.D. \& Walberg, H.J. (1993). Toward a knowledge base for school learning. Review of Educational Research, 63 (3), 249- 294. https://doi.org/10.3102/00346543063003249

Webster, R., Blatchford, P., Bassett, P., Brown, P., Martin, C. \& Russell, A. (2010). Double stan- dards and first principles: Framing teaching assistant support for pupils with special educational needs. European Journal of Special Needs Education, 25(4), 319-336. https://doi.org/10. $1080 / 08856257.2010 .513533$

Wohlgemuth, K. (2009). Schulbegleitung in Thüringen - Rahmenbedingungen, Aufgaben und Belastungen. Unveröffentlichte Diplomarbeit. Jena: Friedrich-Schiller-Universität.

Yusuf, S., Wittes, J., Probstfield, J. \& Tyroler, H. A. (1991). Analysis and interpretation of treatment effects in subgroups of patients in randomized clinical trials. Jama, 266(1), 93-98.

Zauner, M. \& Zwosta, M. (2014). Effektestudie zu Schulbegleitungen. Abgerufen am 22.4. 2018 von https://www.kjf-regensburg.de/documents/ 10502/1053779/Effektestudie 10.11.14.pdf/38f 5880c-eag6-4aff-895f-b298bf59d6c9

\section{Anschrift des Autors}

\section{Florian Schindler \\ TU Dortmund}

Fakultät Rehabilitationswissenschaften Fachgebiet Rehabilitation und Pädagogik bei Lernbehinderungen

Emil-Figge-Straße 50

D-44227 Dortmund

E-Mail: florian.schindler@tu-dortmund.de 